May 4, 1999

LBNL-43104

UCB-PTH-99/15

hep-ph/9904399

\title{
Seasonal Variations of the ${ }^{7}$ Be Solar Neutrino Flux円
}

\author{
André de Gouvêa, Alexander Friedland, and Hitoshi Murayama \\ Theoretical Physics Group \\ Ernest Orlando Lawrence Berkeley National Laboratory \\ University of California, Berkeley, California 94720 \\ and \\ Department of Physics \\ University of California, Berkeley, California 94720
}

\begin{abstract}
Measuring the ${ }^{7} \mathrm{Be}$ solar neutrino flux is crucial towards solving the solar neutrino puzzle. The Borexino experiment, and possibly the KamLAND experiment, will be capable of studying the ${ }^{7}$ Be neutrinos in the near future. We discuss (1) how the seasonal variation of the Borexino and KamLAND data can be used to measure the ${ }^{7} \mathrm{Be}$ solar neutrino flux in a background independent way and (2) how anomalous seasonal variations might be used to discover vacuum neutrino oscillations, independent of the solar model and the measurement of the background. In particular, we find that, after three years of Borexino or KamLAND running, vacuum neutrino oscillations can be either established or excluded for almost all values of $\left(\sin ^{2} 2 \theta, \Delta m^{2}\right)$ preferred by the Homestake, GALLEX, SAGE, and Super-Kamiokande data. We also discuss how well seasonal variations of the data can be used to measure $\left(\sin ^{2} 2 \theta, \Delta m^{2}\right)$ in the case of vacuum oscillations.
\end{abstract}

*This work was supported in part by the U.S. Department of Energy under Contracts DE-AC03-76SF00098, in part by the National Science Foundation under grant PHY-9514797. HM was also supported by the Alfred P. Sloan Foundation and AdG by CNPq (Brazil). 


\section{Introduction}

The question whether neutrinos have non-zero mass has been an outstanding issue in particle physics for many decades. Recently there have been new exciting developments in the indirect search for neutrino masses via neutrino oscillations. Major progress has been achieved by studying atmospheric neutrinos, culminating in the announcement of evidence for muon neutrino oscillations by the Super-Kamiokande collaboration [1]. The most striking signal presented in [1] is the up-down asymmetry of the atmospheric muon neutrino flux. The choice of this particular quantity eliminates many theoretical uncertainties and the final result is very robust. In fact, at present time, this result represents perhaps the best evidence for physics beyond the Standard Model.

Another very active area of research is the study of neutrinos coming from the Sun. Ever since the Homestake experiment [2] reported its first results, there has been disagreement between theoretical predictions and measurements of the solar neutrino flux. For many years, however, it was not possible to determine if the observed discrepancy was due to problems with the experiment and/or with the modeling of the Sun, or if it was, in fact, a sign of new physics. In the last decade other neutrino experiments, Kamiokande [3], GALLEX [4], SAGE [5], and more recently Super-Kamiokande [6], have also measured the solar neutrino flux, with different energy thresholds and using very different techniques. All four experiments confirm a deficit in the observed number of solar neutrino induced events. Moreover, it has recently become clear that it is virtually impossible to concoct a solar model which would fit all the data [7, 8]. On the other hand, the results of all experiments can be explained by assuming that the electron neutrino oscillates into a different flavor state.

There are two neutrino oscillation scenarios that are capable of faithfully explaining the solar neutrino data [7]. One scenario makes use of the MSW effect [9, 10], where the electron neutrino conversion into another neutrino flavor is due to flavor dependent interactions with solar matter. The other scenario assumes that the neutrino oscillation length is comparable to the Earth-Sun distance, and simple vacuum oscillations are sufficient. This scenario is also known as the "just-so" solution [11]. Both scenarios allow the electron neutrinos to oscillate into other active species or sterile neutrinos.

The solar neutrino energy spectrum is determined by several nuclear reactions which take place in the Sun's core [12], and different experiments 
are sensitive to neutrinos produced by different nuclear reactions. SuperKamiokande, for example, a very large water Cherenkov detector, is currently sensitive to solar neutrinos with energies slightly above $5.5 \mathrm{MeV}$. Almost all neutrinos it detects come from the decay of ${ }^{8} \mathrm{~B}\left({ }^{8} \mathrm{~B} \rightarrow{ }^{8} \mathrm{Be}^{*}+e^{+}+\nu_{e}\right)$. .

Another solar reaction that gives rise to neutrinos is the process of electron capture by a ${ }^{7} \mathrm{Be}$ nucleus $\left({ }^{7} \mathrm{Be}+e^{-} \rightarrow{ }^{7} \mathrm{Li}+\nu_{e}\right)$. Neutrinos from this reaction have energies below the Super-Kamiokande threshold, but are accessible to the radiochemical experiments Homestake, GALLEX, and SAGE. If one naively assumes that the suppression in the neutrino flux is due to the suppression of individual neutrino sources $\left({ }^{8} \mathrm{~B},{ }^{7} \mathrm{Be}\right.$, etc $)$ in the Sun, the combination of the Super-Kamiokande data with that of the radiochemical experiments indicates that the flux of ${ }^{7}$ Be neutrinos is virtually absent [8, 13] (the best fit value of the ${ }^{7} \mathrm{Be}$ flux is in fact negative!). In the case of neutrino oscillations, all solutions to the solar neutrino puzzle indicate that the ${ }^{7} \mathrm{Be}$ neutrino flux is suppressed, in some cases very strongly. Thus, at present, there is great demand for experiments that would accurately measure the flux of the ${ }^{7}$ Be neutrinos. Two upcoming experiments, Borexino and KamLAND, may have the capability to do exactly that.

In this paper, we present a quantitative study of what can be accomplished by measuring the seasonal variations of the ${ }^{7} \mathrm{Be}$ neutrino flux at Borexino and KamLAND. Seasonal variations of the solar neutrino flux are of course expected, due to the Earth's eccentric orbit. The number of neutrinos of all flavors reaching the Earth is larger when the Earth is closer to the Sun than when it is farther away, and should vary as $1 / L^{2}$. In the case of no neutrino oscillations or of the MSW solution to the solar neutrino puzzle, the number of ${ }^{7} \mathrm{Be}$ solar neutrino induced events is supposed to vary according to the $1 / L^{2}$ law, following the variation of the total neutrino flux. This will be referred to as the "normal" seasonal variation.

If vacuum oscillations are the solution to the solar neutrino puzzle, large, anomalous seasonal variations of the number of ${ }^{7} \mathrm{Be}$ solar neutrino induced events might be detected [11, 14]. It is well known that neutrino oscillation effects depend on the distance to the neutrino source, and different EarthSun distances may yield very different $\nu_{e}$ survival probabilities [15, 16. The anomalous seasonal variation effect should be more pronounced in ${ }^{7} \mathrm{Be}$ neutrinos than in ${ }^{8} \mathrm{~B}$ neutrinos (the latter was recently studied in [17]). This is

*There is a small fraction of the neutrinos that can be detected at Super-Kamiokande coming from the reaction ${ }^{3} \mathrm{He}+p \rightarrow{ }^{4} \mathrm{He}+e^{+}+\nu_{e}$. 
due to one important feature which distinguishes ${ }^{7} \mathrm{Be}$ neutrinos from ${ }^{8} \mathrm{~B}$ and other abundant types of solar neutrinos: because they are produced as part of a two-body final state, the neutrino energy spectrum is mono-energetic." The details will become clear when we discuss the anomalous seasonal variation effect, in Sec. 3 .

In the case of no anomalous seasonal variations, if one has enough statistics and a small enough background, the time variation of the data can be used to measure the solar neutrino flux, given that the number of background events is constant in time. We will analyze how well Borexino and KamLAND can perform this type of measurement. We are particularly interested in analyzing the relevance of this technique when the number of electron neutrinos reaching the detector is very suppressed with respect to the Standard Solar Model predictions, as might be the case if there are $\nu_{e} \rightarrow \nu_{\mu, \tau}$ oscillations for the small angle MSW solution.

The paper is organized as follows. In Sec. 2 we discuss how seasonal variations might be used to determine the solar neutrino flux at Borexino and KamLAND, in such a way that no separate measurement of the number of background events is required. In Sec. 3 we analyze the effect of the vacuum oscillation solutions to the solar neutrino puzzle on the annual variation of the number of detected events at Borexino and KamLAND. In particular we describe the region of the $\left(\sin ^{2} 2 \theta, \Delta m^{2}\right)$ parameter space where vacuum oscillations can be discovered by studying the seasonal variations of the data. In Secs. 1 and 5 we describe how the measurement of the seasonal variation of the ${ }^{7} \mathrm{Be}$ solar neutrino flux may be used to either measure the neutrino oscillation parameters, $\sin ^{2} 2 \theta$ and $\Delta m^{2}$, or exclude a large portion of the $\left(\sin ^{2} 2 \theta, \Delta m^{2}\right)$ parameter space. In Sec. 6 we summarize our results and conclude.

\footnotetext{
${ }^{\dagger}$ In fact there are two distinct neutrino energies, 0.383 and $0.862 \mathrm{MeV}$, corresponding to different final states of the ${ }^{7} \mathrm{Li}$ nucleus. Borexino and KamLAND are only sensitive to the higher energy component.

$¥$ Actually, a time-dependent background is also acceptable, as long as it can be monitored and understood well enough.

${ }^{\S}$ If $\nu_{e}$ oscillates into sterile neutrinos, the suppression is even more pronounced, due to the absence of neutral current $\nu_{\mu, \tau^{-}} e$ elastic scattering. We do not consider oscillations into sterile neutrinos in this paper.
} 


\section{Measuring the ${ }^{7} \mathrm{Be}$ Solar Neutrino Flux}

As was already pointed out, measuring the flux of ${ }^{7} \mathrm{Be}$ neutrinos is crucial towards understanding the solar neutrino puzzle. Borexino [18 plans to do this measurement by using 300 tons of organic liquid scintillator to detect recoil electrons from elastic $\nu$-e scattering. Since the scintillator has no directional information, and the signal is characterized only by the scintillation light produced by the recoil electron, the background has to be kept under control. This places a very stringent constraint on the radio-purity of the scintillator and on the activity of the material in the detector. Borexino anticipates 100 tons of fiducial volume for detecting solar neutrinos.

KamLAND [19], which was originally conceived as a reactor neutrino experiment with an unprecedented baseline $(170 \mathrm{~km}$ on the average), may also be able to study ${ }^{7}$ Be solar neutrinos, if rigorous yet attainable requirements on the radio-purity and activity are met. We assume throughout the paper that KamLAND will use 600 tons of fiducial volume for detecting solar neutrinos (the size of the fiducial volume will depend on the background rate, which is currently unknown). We concentrate our analysis on Borexino, which is an approved dedicated solar neutrino experiment, and discuss KamLAND, whose uses for solar neutrino studies are at present being proposed [20], as a possible higher statistics improvement.

It is important to define what is meant by "measuring the ${ }^{7}$ Be solar neutrino flux." In reality, what the experiments are capable of measuring is the number of recoil electrons induced by solar neutrino interactions in a given recoil electron kinetic energy range (kinematic range). This information can only be converted into a solar neutrino flux measurement if one knows the flavor composition of the solar neutrinos [21]. Explicitly, assuming that the solar neutrino flux is composed of $\nu_{e}$ (with fraction $P$ ) and $\nu_{\mu, \tau}$ (with fraction $Q=1-P$ ),

$$
\text { \#recoil electrons } / \text { time }=\Phi \times\left(P \sigma_{\nu_{e}-e}+(1-P) \sigma_{\nu_{\mu, \tau^{-}}}\right) N_{e}
$$

where $\Phi$ is the neutrino flux, $N_{e}$ is the number of target electrons, and

$$
\sigma_{\nu_{x}-e} \equiv \int_{T_{\min }}^{T_{\max }} d T\left(\frac{\mathrm{d} \sigma}{\mathrm{d} T}\right)_{\nu_{x^{-}} e}
$$

with $\left(\frac{\mathrm{d} \sigma}{\mathrm{d} T}\right)_{\nu_{x}-e}$ being the differential cross section for $\nu_{x^{-}} e$ scattering for a given kinetic energy $T$ of the recoil electron. $T_{\min }$ and $T_{\max }$ define the kinematic 
range. In the case of neutrino oscillations, $P$ is the the survival probability for electron neutrinos, while $1-P$ is the probability that $\nu_{e}$ will oscillate into $\nu_{\mu, \tau}$.

If the flavor composition of the flux is not known, all that can be quoted is the effective neutrino flux, $\Phi_{\text {eff }}$, which is calculated from the number of measured recoil electrons assuming that there are only electron neutrinos coming from the Sun. Explicitly,

$$
\Phi_{\text {eff }} \equiv \frac{\# \text { recoil electrons } / \text { time }}{\sigma_{\nu_{e}-e} N_{e}}=\Phi \times\left(P+(1-P) \frac{\sigma_{\nu_{\mu, \tau}-e}}{\sigma_{\nu_{e}-e}}\right) .
$$

Clearly, if $P=1, \Phi_{\text {eff }}=\Phi$. It is important to remember that $\sigma_{\nu_{\mu, \tau^{-}}} / \sigma_{\nu_{e}-e}<1$ and therefore $\Phi_{\text {eff }} \leq \Phi$. The ratio of the neutrino elastic cross sections depends on the energy of the incoming neutrino and the kinematic range to which each particular experiment is sensitive. For $E_{\nu}=0.862 \mathrm{MeV}$ and the Borexino (KamLAND) kinematic range 250-800 keV (280-800 keV), $\sigma_{\nu_{\mu, \tau^{-}} e} / \sigma_{\nu_{e^{-}}}=0.213(0.214)$. It is this effective electron neutrino flux, $\Phi_{\text {eff }}$, that is referred to, throughout this paper (and in general), as the ${ }^{7} \mathrm{Be}$ solar neutrino flux.

In order to determine the number of recoil electrons induced by solar neutrino interactions, it is crucial to determine the number of background events. The number of background events can be estimated by various techniques, which we do not address in this paper. It is worthwhile to point out, however, that this is a very difficult process and it would be highly desirable to have an independent way to determine the ${ }^{7}$ Be solar neutrino flux in order to make the final results more convincing. This may be possible if one looks at the seasonal variation of the number of detected events.

In the following, we study the seasonal variation of the event rate as a means to measure the ${ }^{7} \mathrm{Be}$ solar neutrino flux. The distance between the Earth and the Sun varies slightly over seasons because of the eccentricity of the Earth's orbit. The perihelion (when the Earth is closest to the Sun) occurs around January first. The eccentricity of the Earth's orbit is $\epsilon=0.017$, and hence the distance varies as

$$
L=L_{0}(1-\epsilon \cos (2 \pi t / \text { year }))
$$

to the first order in $\epsilon$. Here, $t$ is the time measured in years from the perihelion, and $L_{0}=1.496 \times 10^{8} \mathrm{~km}$ is one astronomical unit. The neutrino flux varies as $1 / L^{2}$ and hence shows a seasonal variation of about $7 \%$ from 
minimum to maximum. The change in the Earth-Sun distance between the aphelion and the perihelion is given by

$$
\Delta L \equiv L_{\max }-L_{\min }=2 \epsilon L_{0}=5.1 \times 10^{6} \mathrm{~km} .
$$

By fitting the event rate to the seasonal variation expected due to the eccentricity,

$$
B+S\left(\frac{L_{0}}{L}\right)^{2}
$$

one can extract the background event rate $B$ and the signal event rate $S$ independently. As long as the detector is monitored well and its performance is sufficiently stable, this method will be only limited by statistics.

Borexino expects 53 events/day [" according to the BP95 [22] Standard Solar Model (SSM), together with 19 background events/day [18], after the statistical subtraction of the known background sources. This is done by pulse shape discrimination against the $\alpha$-particle background and the measurement of Bi-Po pairs via $\alpha-\beta$ coincidence. This in turn allows the statistical subtraction of processes in the ${ }^{238} \mathrm{U}$ and ${ }^{232} \mathrm{Th}$ chains which are in equilibrium. It is also assumed that the experiment can achieve a radiopurity of $10^{-16} \mathrm{~g} / \mathrm{g}$ for $\mathrm{U} / \mathrm{Th}, 10^{-18} \mathrm{~g} / \mathrm{g}$ for ${ }^{40} \mathrm{~K},{ }^{14} \mathrm{C} /{ }^{12} \mathrm{C}=10^{-18}$, and no $\mathrm{Rn}$ diffusion. For KamLAND we use 466 events/kt/day for the signal and 217 events/kt/day [20] for the background under similar assumptions but with larger cosmogenic background (especially ${ }^{11} \mathrm{C}$ ) and some Rn diffusion. Assuming 600 t of fiducial volume, we expect 280 signal events/day and 130 background events/day. Throughout the paper, we will assume that the number of background events is either constant in time or its time dependence is sufficiently well understood by monitoring. We neglect systematic effects and assume that there are only statistical uncertainties.

Under these assumptions, Fig. 1 depicts a simulation of the seasonal variation of the "data" for both Borexino and KamLAND, after three years of running. The plots are for the case of the small angle MSW solution to the solar neutrino puzzle, where the $\nu_{e}$ 's produced by ${ }^{7}$ Be electron capture inside the Sun have almost completely oscillated into $\nu_{\mu}$ or $\nu_{\tau}$, and the event rate is reduced to $21.3 \%(21.4 \%)$ of the SSM prediction at Borexino (KamLAND).

\footnotetext{
${ }^{9}$ For simplicity, we neglect the contribution of solar neutrino sources other than ${ }^{7} \mathrm{Be}$ electron capture throughout the paper. In particular we neglect the contribution of neutrinos produced in the $\mathrm{CNO}$ cycle, which is about $10 \%$ of that from the ${ }^{7} \mathrm{Be}$ neutrinos.
} 

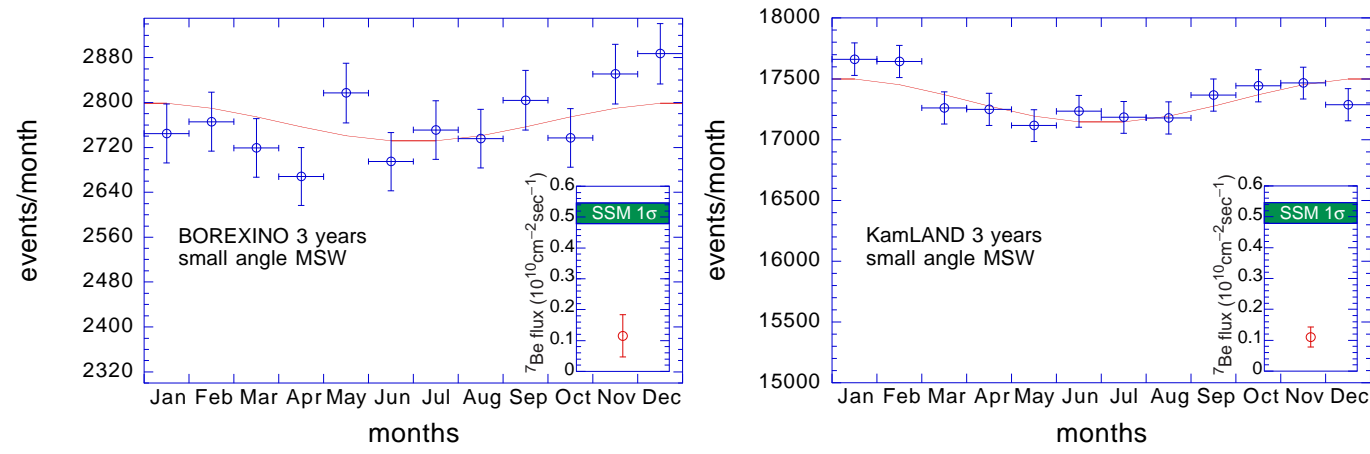

Figure 1: The simulated seasonal variation of the ${ }^{7} \mathrm{Be}$ flux for the case of the small angle MSW solution, for three years of Borexino (left) and KamLAND (right) running. The inset shows the measured flux of ${ }^{7} \mathrm{Be}$ neutrinos from the fit to the seasonal variation of the event rate (point with error bar) and the SSM prediction (shaded band).

In the fit to the data, both the background and the ${ }^{7}$ Be flux are allowed to float.

This analysis can be repeated for different values of the ${ }^{7} \mathrm{Be}$ flux, or, equivalently, for different survival probabilities for $\nu_{e}$. Fig. 2 depicts the expected $1 \sigma$ statistical accuracy of the ${ }^{7}$ Be flux measurement, together with the central value normalized by the SSM prediction, as a function of the survival probability for $\nu_{e}$. We emphasize that this measurement technique assumes no knowledge of the background.

The important information one should obtain from this analysis is if one can indeed measure a nonzero ${ }^{7}$ Be solar neutrino flux. For example, in the case of the small angle MSW solution, the $\nu_{e}$ survival probability is very close to zero and, assuming the expected number of background events, Borexino's measured neutrino flux is less than $1.5 \sigma$ away from zero. The situation at KamLAND is much better, and in the case of the small angle MSW solution a healthy 3 sigma-away-from-zero measurement of the flux is obtained, if the background is as low as expected. The significance of the measured flux increases for larger survival probabilities, as in the case of the large angle and the low $\Delta m^{2}$ MSW solutions.

A similar analysis can be performed in order to determine how many background events each experiment can tolerate in order to claim a solar neutrino flux measurement which is $3 \sigma$ away from zero. Fig. 3 depicts the 

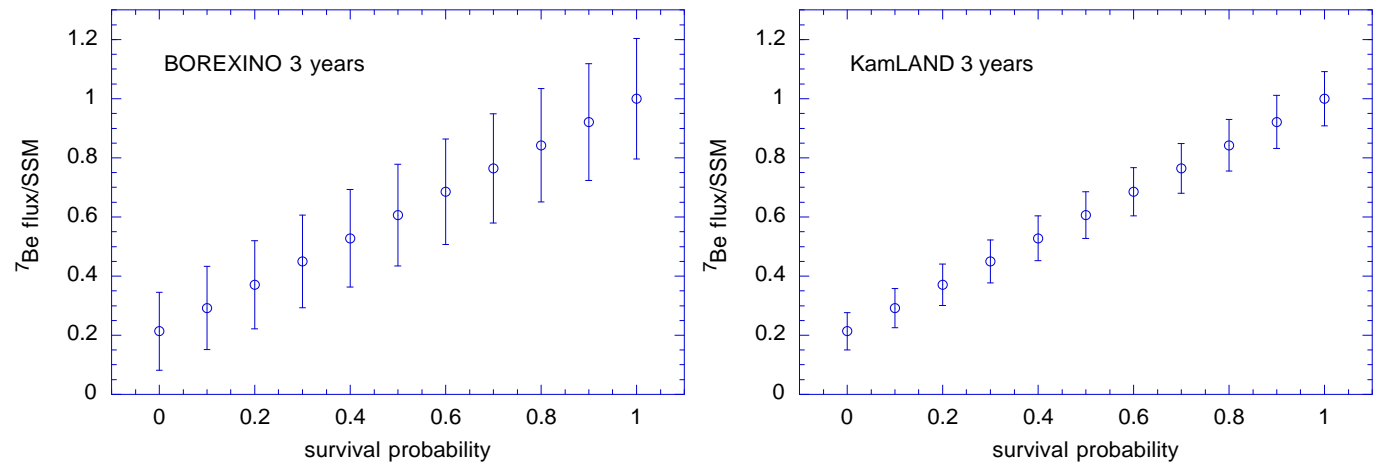

Figure 2: The expected $1 \sigma$ statistical accuracy of the ${ }^{7}$ Be neutrino flux measurement, together with the central value normalized by the flux predicted by the SSM, as a function of the $\nu_{e}$ survival probability at Borexino (left) and KamLAND (right), after three years of data taking.
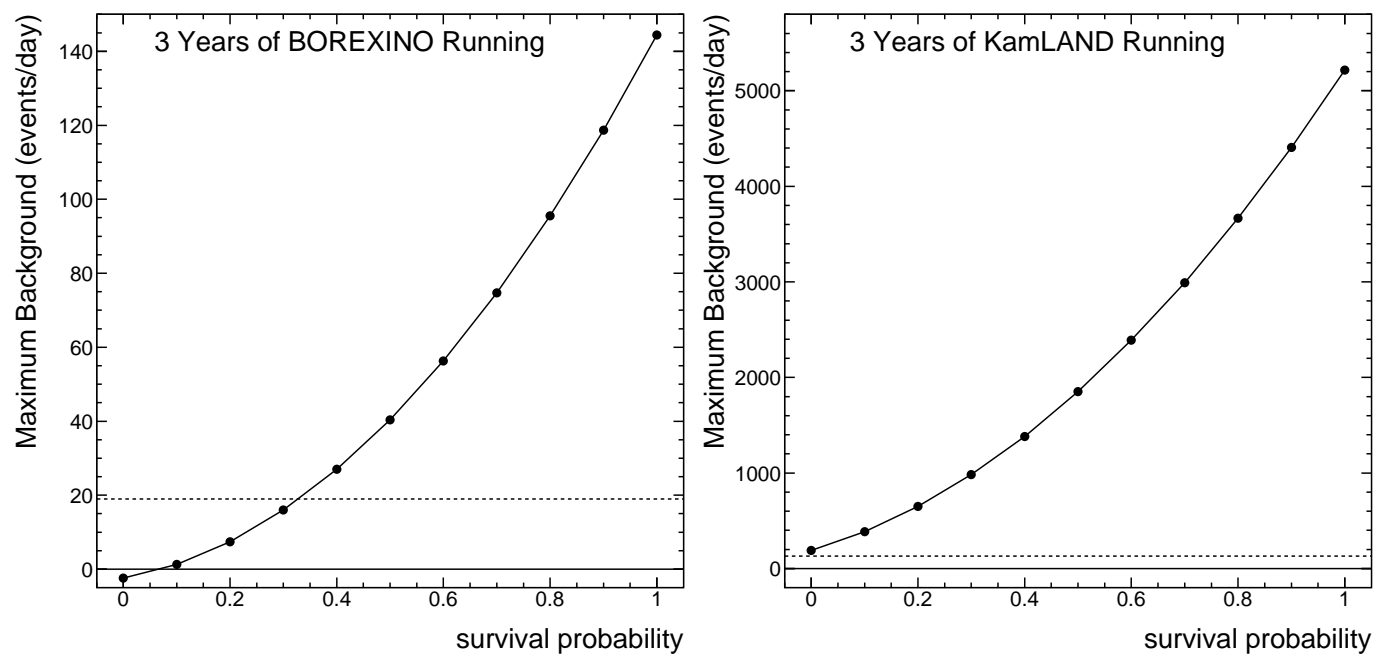

Figure 3: The maximum number of background events allowed per day at Borexino (left) or KamLAND (right), for 3 years of running, in order to measure a solar neutrino flux which is $3 \sigma$ away from zero. The dashed lines indicate the currently anticipated number of background events per day. 
maximum number of background events per day allowed for 3 years of Borexino or KamLAND running. It is worthwhile to comment that, in the case of Borexino and the small angle MSW solution $(P \simeq 0)$, a 3 sigma-awayfrom-zero measurement of the neutrino flux is not attainable in three years, even in the case of no background (note that for $P \lesssim 0.05$ the required maximum background to achieve a three $\sigma$ measurement of the flux is negative, i.e., impossible to achieve). Therefore, for Borexino, this simple, background independent analysis using the seasonal variation of the data is not particularly powerful in the case of the small angle MSW solution, due to statistical limitations.

\section{Sensitivity to Vacuum Oscillations}

In this section we study the discovery potential of the Borexino and KamLAND experiments in the region of $\Delta m^{2}$ corresponding to the vacuum oscillation solution to the solar neutrino problem. In this case, the pattern of seasonal variations can be very distinct from the normal pattern discussed in the previous section.

The basic idea is the following. The survival probability $P$ for an electron neutrino in the case of neutrino vacuum oscillations between two flavor states ${ }^{\circledast}$ is given by

$$
P=1-\sin ^{2} 2 \theta \sin ^{2}\left(1.27 \Delta m^{2} \frac{L}{E}\right),
$$

where the neutrino energy $E$ is in $\mathrm{GeV}$, the distance $L$ in $\mathrm{km}$, and the difference of masses-squared $\Delta m^{2}$ in $\mathrm{eV}^{2}$. Model-independent analyses of all solar neutrino data show the need for an energy-dependent suppression of the $\nu_{e}$ flux. The "just-so" solution achieves this by choosing $\Delta m^{2}$ such that the corresponding neutrino oscillation length

$$
L_{\mathrm{osc}} \equiv \frac{\pi E}{1.27 \Delta m^{2}}=2.47 \times 10^{8} \mathrm{~km} \times\left(\frac{E}{10 \mathrm{MeV}}\right)\left(\frac{10^{-10} \mathrm{eV}^{2}}{\Delta m^{2}}\right)
$$

is of the order of one Astronomical Unit ( $1 \mathrm{a} . \mathrm{u} .=1.496 \times 10^{8} \mathrm{~km}$ ); hence the name "just-so". More specifically, the oscillation length is assumed comparable to 1 a.u. for ${ }^{8} \mathrm{~B}$ neutrinos $\left(E_{\nu} \approx 10 \mathrm{MeV}\right)$; at the same time, the

\footnotetext{
*One can assume the more complicated case of oscillations between three neutrino flavor states. In this paper we limit our studies to the case of oscillations between two flavor states.
} 

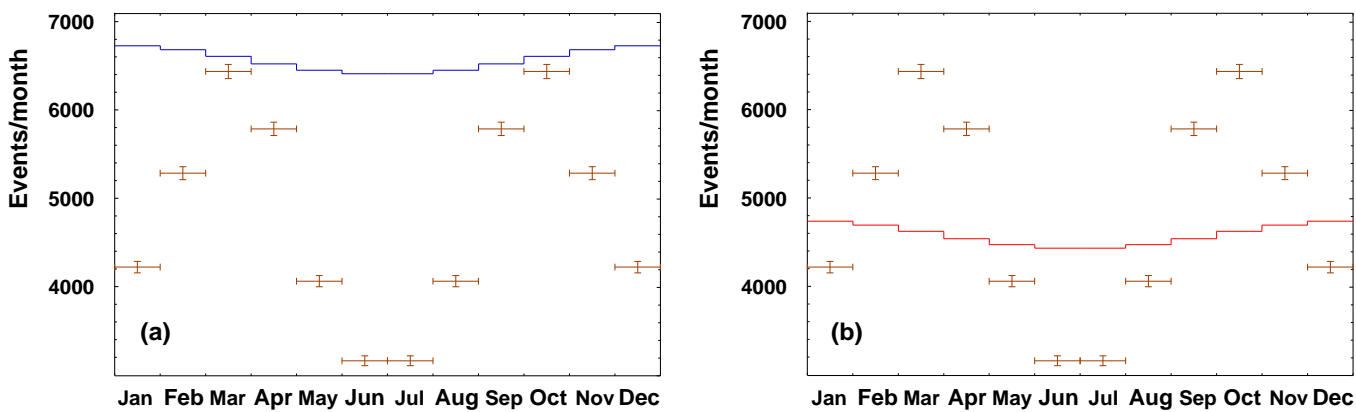

Figure 4: Illustration of the effect of vacuum oscillations on the shape of the seasonal variation of the solar neutrino data. The points with statistical error bars represent the number of events/month expected at Borexino after 3 years of running for $\Delta m^{2}=3 \times 10^{-10} \mathrm{eV}^{2}, \sin ^{2} 2 \theta=1$. The histogram in (a) shows the number of events predicted by the SSM without neutrino oscillations, plus the number of anticipated background events. The histogram in (b) shows the same quantity after adjusting the solar neutrino flux and the background rate so as to minimize the value of $\chi^{2}$, as explained in the text. The difference between the case with oscillations and the one without oscillations is still apparent.

oscillation length of ${ }^{7} \mathrm{Be}$ neutrinos $\left(E_{\nu}=0.862 \mathrm{MeV}\right)$ is an order of magnitude smaller and, for sufficiently large $\Delta m^{2}$, can be comparable to the seasonal variation of the Earth-Sun distance due to the eccentricity of the Earth's orbit, $\Delta L$ (see Eq. (2.5)). As a consequence, the flux of ${ }^{7}$ Be neutrinos detected on the Earth may exhibit an anomalous seasonal variation, beyond the normal $1 / L^{2}$ effect discussed in the previous section.

Such anomalous variation could serve as a unique signature of vacuum oscillations [11, 14]. Moreover, as we will show in this section, both Borexino and KamLAND will be able to cover a large portion of the "just-so" parameter space, even without relying on a particular solar model or estimate of the background rate, just by analyzing the shape of their data. In this sense the discovery of an anomalous seasonal variation at one of these experiments would be as robust a result as the Super-Kamiokande measurement of the up-down asymmetry for the atmospheric muon neutrinos.

To illustrate the main idea, we choose a particular point $\left(\Delta m^{2}=3 \times\right.$ $10^{-10} \mathrm{eV}^{2}, \sin ^{2} 2 \theta=1$ ) in the allowed region of the "just-so" parameter 
spacell and compute the corresponding seasonal distribution of the neutrino events at Borexino after 3 years of running. We use the number of background events and the expected number of signal events (before the effect of neutrino oscillations) quoted in Sec. 2. The results are shown in Fig. 1 by the set of "data" points with error bars; each point represents the number of events expected in a given month and the vertical error bars show the corresponding statistical uncertainties. The histogram in Fig. 团(a) shows "theoretical" event rates expected for non-oscillating neutrinos, provided the background rate is known accurately and the SSM prediction for the neutrino flux is trusted. One can see that under these assumptions vacuum neutrino oscillations with $\Delta m^{2}=3 \times 10^{-10} \mathrm{eV}^{2}, \sin ^{2} 2 \theta=1$ would be trivial to discover.

More importantly, the experiment would be able to claim the discovery even without relying on an estimate of the background rate or the value of incoming neutrino flux predicted by the SSM. It is intuitively obvious from the figure that the vacuum oscillation "data" points cannot be fit by the "theoretical" curve even if the background and the solar neutrino flux are varied freely, unless one assumes neutrino oscillations. This can be quantified as follows. For a given background rate $b$ and signal event rate $s$, we define the $\chi^{2}$ value of the fit for an "average" experiment:

$$
\chi^{2}(s, b)=N_{\text {d.o.f. }}+\sum_{i}^{N_{\text {bins }}} \frac{\left(d_{i}-b-s \cdot h_{i}\right)^{2}}{d_{i}},
$$

where $N_{\text {bins }}$ is the number of bins, $N_{\text {d.o.f. }}$ is the number of degrees of freedom, $d_{i}$ is the average expected number of neutrino events in the $i$ th bin, and $h_{i}$ is given by $h_{i}=\int_{i-1}^{i}\left(1-\epsilon \cos \left(2 \pi x / N_{\text {bins }}\right)\right)^{2} d x$. The constant term $N_{\text {d.o.f. in }}$ Eq. (3.3) is added to take into account the effect of statistical fluctuations in the data. In a single experiment, statistical fluctuations make the number of neutrino events in the $i$ th bin slightly different from $d_{i}$, and $\chi^{2}$ is computed by an expression similar to Eq. (3.3), with $d_{i}$ replaced by the number of events measured in the $i$ th bin and without the constant term, $N_{\text {d.o.f. }}$ In our analysis, however, we are interested in the sensitivity of an "average" experiment. As proven in Appendix A, averaging over many experiments results in the definition of $\chi^{2}$ given in Eq. (3.3), with the constant term $N_{\text {d.o.f. }}$. This agrees with the conventional wisdom that, if a function describes data correctly, the average expected value of $\chi^{2}$ should be equal to the number of

${ }^{\dagger}$ Based on the analysis of the total rates in the Homestake, GALLEX, SAGE, and Super-Kamiokande experiments. See Fig. 5 in [7]. 
degrees of freedom. Given this definition, we can choose values of $s$ and $b$ that minimize the $\chi^{2}$; the only restriction imposed is that both $s$ and $b$ be non-negative. For the case at hand the minimum occurs when $b$ is zero and $s$ is 0.95 times the SSM prediction (see Fig. 四(b)). As expected, even after this change the "data" points and the histogram are very different. (Numerically, $\chi^{2}=2935$ which for 10 degrees of freedom implies a confidence level of $1-9 \times 10^{-626}$ !). 周

We now extend this approach, and scan the entire $\left(\sin ^{2} 2 \theta, \Delta m^{2}\right)$ plane (for an earlier work with a more simplified analysis which does not consider the presence of background, see [15]). In the analysis below, we follow the same steps as before: the "data" is simulated according to the expected number of background and signal events, plus the effect of neutrino oscillations, for each value of $\left(\sin ^{2} 2 \theta, \Delta m^{2}\right)$, binned into a certain number of bins $N_{\text {bins }}$, and then compared to the "theoretical" predictions for the case of no oscillations. The $\chi^{2}$ is computed according to Eq. (3.3) and minimized with respect to both the signal $(s)$ and background $(b)$. The confidence level (CL) corresponding to the minimal value of $\chi^{2}$ and $N_{\text {d.o.f. }}=N_{\text {bins }}-2$ degrees of freedom is then determined, and the region in which the CL is less than a given number is isolated. This case, when both the number of background events and the incoming solar neutrino flux are considered unknown in the fit, is the most conservative one, and yields the smallest sensitivity region. Later we also study less conservative cases, where we assume in the "data" analysis that the incoming neutrino flux is the one predicted by the SSM and/or that the background rate is known.

We now apply this most conservative procedure to study the experimental reach of Borexino after 3 years of operation. In Figure 5 we show the results of the scan for $95 \%$ and $5 \sigma$ CL. As one can see from the figure, even at $5 \sigma$ CL a large portion of the parameter space above $\Delta m^{2} \sim 10^{-10} \mathrm{eV}^{2}$ is covered (white region). In this region the neutrino oscillation length $L_{\mathrm{osc}}$ is smaller than the seasonal variation of the Earth-Sun distance $\Delta L$. On the other hand, below $\Delta m^{2} \sim 10^{-10} \mathrm{eV}^{2}$ one can see a series of spikes protruding through the sensitivity region. It is important to understand the origin of these spikes. Since we adjust the level of signal and background in the fit, we are not sensitive to the absolute event rate, only to its variation during the year. For $\Delta m^{2} \lesssim 10^{-10} \mathrm{eV}^{2}$ the oscillation length is larger than $\Delta L$ and the

‡This number is, of course, unrealistic, and the true confidence level in this case will be dominated by systematic effects. 


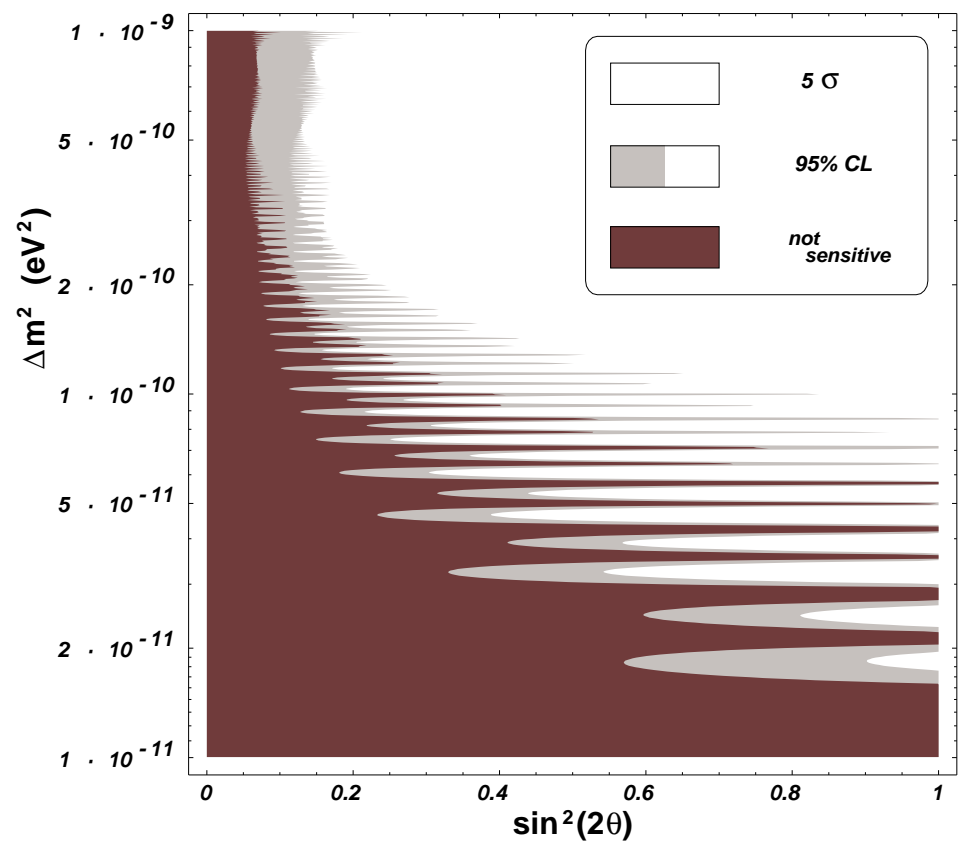

Figure 5: The sensitivity region of the Borexino experiment in 3 years, if the analysis does not assume any knowledge of the background rate or the incoming solar neutrino flux. In the unshaded region the "data" is at least $5 \sigma$ away from the best no-oscillations fit. In the lightly shaded region the discrepancy is greater than $95 \%$ CL but less than $5 \sigma$ CL.

amplitude of the variation of the event rate is roughly proportional to the first derivative of Eq. (3.1) with respect to $L$. In the regions where this derivative nearly vanishes, the amplitude of the variations is small and the signal is indistinguishable from the case of no oscillations. This explains why the loss of sensitivity occurs not only when the neutrinos undergo approximately an integer number of oscillations as they travel to the Earth $\left(\Delta m^{2}=n \times 0.143 \times\right.$ $10^{-10} \mathrm{eV}^{2}$ ), but also when the number of oscillations is close to a half-integer $\left(\Delta m^{2}=(n+1 / 2) \times 0.143 \times 10^{-10} \mathrm{eV}^{2}\right)$. In the latter case the absolute neutrino flux is maximally suppressed, but the magnitude of the seasonal variation is small. $\beta^{3}$

Given this explanation, one would expect that the spikes corresponding to

\footnotetext{
${ }^{\S}$ Notice that the regions preferred from the global fits have the absolute ${ }^{7} \mathrm{Be}$ neutrino flux suppressed. See Figs. 9 and 10.
} 
a half-integer number of oscillations should become shorter if in the analysis we choose to rely on the SSM prediction of the incoming neutrino flux and/or on the anticipated background rate. It is straightforward to incorporate the knowledge of both quantities and their uncertainties in our procedure. For example, to impose the value of the incoming neutrino flux predicted by the SSM, we modify the expression of $\chi^{2}$ in Eq. (3.3) by adding an extra term:

$$
\chi^{2}(s, b) \longrightarrow \chi^{2}(s, b)+\frac{\left(s-s_{0}\right)^{2}}{\sigma_{s_{0}}^{2}}
$$

where $s$ and $b$ are the values of the signal and background with respect to which we later minimize $\chi^{2}, s_{0}$ is the SSM prediction for the signal, and $\sigma_{s_{0}}$ is the uncertainty in $s_{0}$. The rest of the analysis is carried out unchanged, except that the number of degrees of freedom is increased by one to $N_{\text {d.o.f. }}=N_{\text {bins }}-1$. To use both the incoming flux predicted by the SSM and the anticipated background rate, two terms are added to Eq. (3.3) and the number of degrees of freedom is increased by two to $N_{\text {d.o.f. }}=N_{\text {bins }}$.

The results of the calculation are shown in Fig. 6. The uncertainty on the solar model prediction of the ${ }^{7}$ Be neutrino flux is taken to be $9 \%$ [23], while the uncertainty on the background is $10 \%$ [20]. As expected, the oddnumbered spikes do become shorter. The one possibility not shown in the plot is the situation when one only assumes knowledge of the background rate. In this case the spikes become significantly thinner, although their length remains virtually unchanged.

In order to extend this analysis to values of $\Delta m^{2}>10^{-9} \mathrm{eV}^{2}$, several issues must be confronted. We will next address these issues one by one, and illustrate the discussion in Fig. 7.

The first and the most obvious point is that the number of bins needs to be changed. The reason is that the frequency of the seasonal variations increases with $\Delta m^{2}$, and above some value $\left(\Delta m^{2} \simeq 8 \times 10^{-10} \mathrm{eV}^{2}\right.$, for 12 bins) integration over the bin size washes out the effect. To avoid this, we change the number of bins from 12 to 365. After the change, the effect of binning kicks in at $\Delta m^{2} \simeq 2.4 \times 10^{-8} \mathrm{eV}^{2}$, as curve 1 in Fig. 7 illustrates.

Next, there are two physical effects one must take into account: one is the interaction of the neutrinos with solar matter (the MSW effect), and the other is the finite width of the ${ }^{7}$ Be solar neutrino line. One may worry about the wash-out of the seasonal variation effect due to the finite size of Sun's core. However, matter effects make the core size effect irrelevant because the 


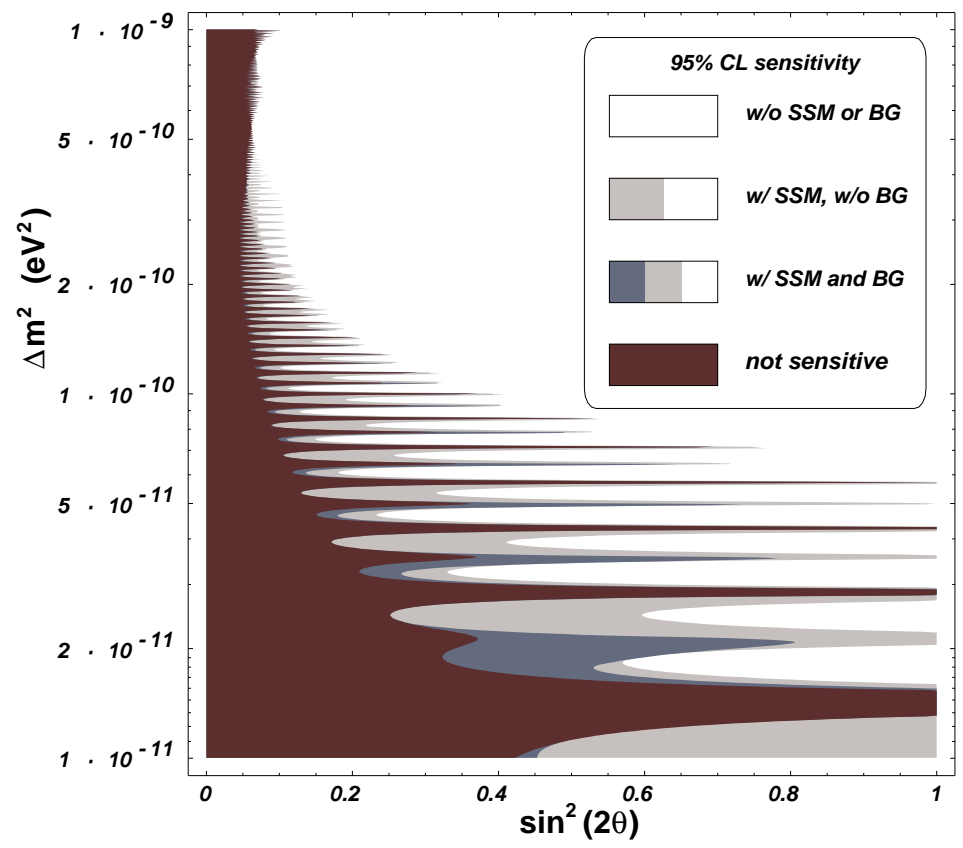

Figure 6: The sensitivity reach of the Borexino experiment after 3 years of running (at 95\% confidence level). The three cases considered are: no knowledge of either the background rate or the incoming solar neutrino flux (the covered region is white); assumption that the incoming solar neutrino flux is the one predicted by the SSM, with $9 \%$ uncertainty (the covered region is white + light gray); assumption that the background rate is known with $10 \%$ uncertainty and the incoming neutrino flux agrees with the SSM, with $9 \%$ uncertainty (the covered region is white + light gray + medium gray)

mixing angle in the Sun's core is small and the oscillations effectively start at the level-crossing point (see Eq. (3.6)). [1]

When a $\nu_{e}$ is created by the electron capture process in the core of the Sun, its Hamiltonian is dominated by the matter effect $\sqrt{2} G_{F} n_{e}\left(n_{e}\right.$ is the electron number density) if $\Delta m^{2} \ll 10^{-5} \mathrm{eV}^{2}$ for ${ }^{7}$ Be neutrinos. We restrict ourselves to $\Delta m^{2}<10^{-7} \mathrm{eV}^{2}$ in the following discussions, as the final sensitivity due to the anomalous seasonal variation is limited by $\lesssim 10^{-8} \mathrm{eV}^{2}$ as will be seen later in this section. Then the mass mixing effect can be completely

\footnotetext{
"We thank E. Lisi and L. Wolfenstein for pointing this out to us. For earlier papers on this particular point, see [9], [1], and in particular, [24].
} 


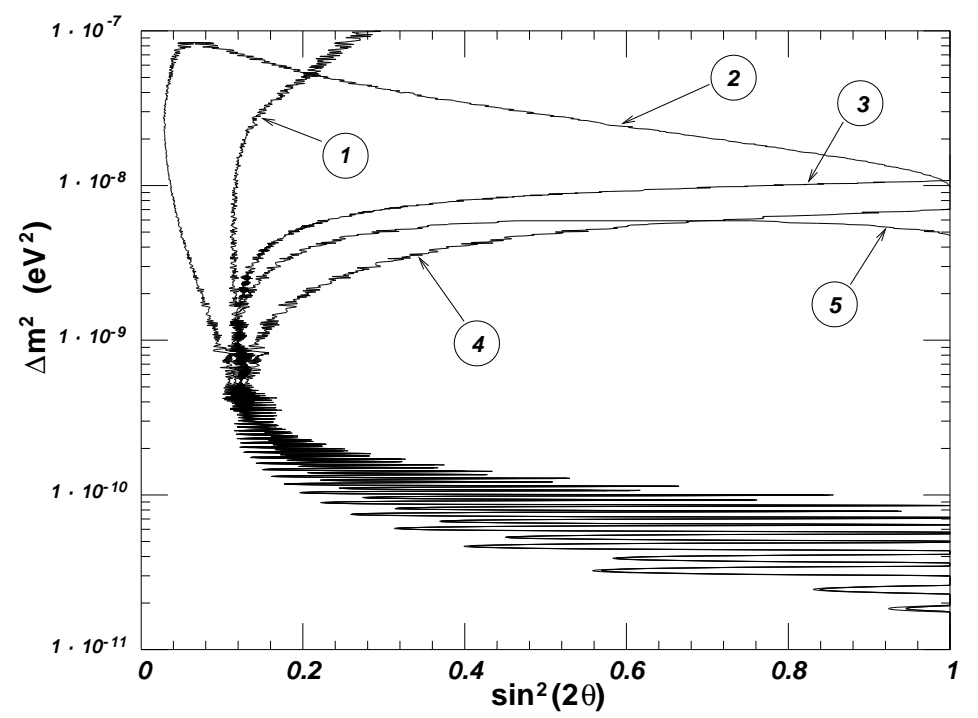

Figure 7: The relative roles of the binning effect, the linewidth effect, and the matter effect, as explained in the text.

ignored at the time of the neutrino production, and one can safely take the produced neutrino to be in a Hamiltonian eigenstate (the one which corresponds to the larger energy in the Sun's core). As it propagates through the Sun, the neutrino follows the instantaneous Hamiltonian eigenstate (in the adiabatic approximation), and exits in the heavier mass eigenstate, $\nu_{2}=$ $\nu_{e} \sin \theta+\nu_{\mu} \cos \theta$. It also has a finite amplitude $A_{c}$ for hopping to the other Hamiltonian eigenstate. The neutrino state that exits the Sun can therefore be written as

$$
\nu_{\text {exit }}=A_{c} \nu_{1}+B_{c} \nu_{2},
$$

with the unitarity constraint $\left|A_{c}\right|^{2}+\left|B_{c}\right|^{2}=1$. Out of the Sun, the two mass eigenstates develop different phases due to the mass difference, $e^{-i \Delta m^{2} t / 2 E_{\nu}}$. Therefore the neutrino state that arrives at the Earth is given by

$$
\nu_{\text {arrival }}=A_{c} \nu_{1}+B_{c} \nu_{2} e^{-i \Delta m^{2} L / 2 E_{\nu}},
$$

up to an overall phase factor. The distance $L$ is between the point of level crossing and the Earth. Finally, the survival probability of the electron neutrino is determined by the $\nu_{e}$ component of $\nu_{\text {arrival }}$, and hence

$$
\begin{aligned}
P & =\left|A_{c} \cos \theta+B_{c} \sin \theta e^{-i \Delta m^{2} L / 2 E_{\nu}}\right|^{2} \\
& =\left|A_{c}\right|^{2} \cos ^{2} \theta+\left|B_{c}\right|^{2} \sin ^{2} \theta+2 \operatorname{Re} A_{c}^{*} B_{c} e^{-i \Delta m^{2} L / 2 E_{\nu}} \sin \theta \cos \theta
\end{aligned}
$$




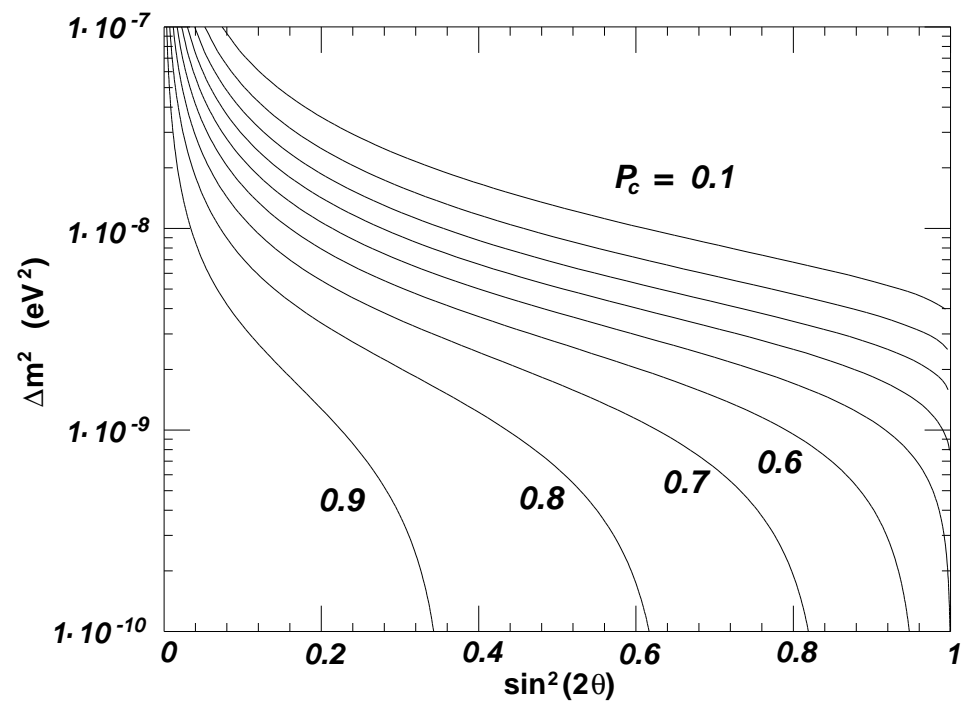

Figure 8: The contour plot of the hopping probability $P_{c}=0.1,0.2, \ldots, 0.9$, for the ${ }^{7}$ Be neutrino energy, using the exponential-profile approximation for the electron number density and Eq. (3.9).

Since $\left|B_{c}\right|^{2}$ is the hopping probability between two Hamiltonian eigenstates in the Sun $P_{c}$, one can rewrite the formula using $P_{c}$ and an additional phase factor $A_{c}^{*} B_{c}=\sqrt{P_{c}\left(1-P_{c}\right)} e^{-i \delta}$,

$$
P=P_{c} \cos ^{2} \theta+\left(1-P_{c}\right) \sin ^{2} \theta+2 \sqrt{P_{c}\left(1-P_{c}\right)} \sin \theta \cos \theta \cos \left(\frac{\Delta m^{2} L}{2 E_{\nu}}+\delta\right) .
$$

An approximate formula for $P_{c}$ was given in [25] using the exponential density profile of the Sun,

$$
P_{c}=\frac{e^{-\gamma \sin ^{2} \theta}-e^{-\gamma}}{1-e^{-\gamma}}
$$

with

$$
\gamma=2 \pi r_{0} \frac{\Delta m^{2}}{2 E_{\nu}}=1.22\left(\frac{\Delta m^{2}}{10^{-9} \mathrm{eV}^{2}}\right)\left(\frac{0.862 \mathrm{MeV}}{E_{\nu}}\right)
$$

where we consider the exponential-profile approximation for the electron number density in the Sun $n_{e} \propto \exp \left(-r / r_{0}\right)$, with $r_{0}=R_{\odot} / 10.54=6.60 \times$ $10^{4} \mathrm{~km}$, given in 26]. Fig. 8 shows the contours of $P_{c}$ on the $\left(\sin ^{2} 2 \theta, \Delta m^{2}\right)$ plane for the ${ }^{7}$ Be neutrino energy $E_{\nu}=0.862 \mathrm{MeV}$. 
The most important consequence of the matter effect is that the vacuum oscillation is suppressed when $P_{c} \rightarrow 0$ (adiabatic limit). The origin of the suppression is simple. When $P_{c}$ is small, the neutrino state that exits the Sun is nearly a pure $\nu_{2}$ state. Since it is a mass eigenstate, only its phase evolves in time and no oscillations take place. The $\nu_{e}$ survival probability then is simply given by the $\nu_{e}$ content of $\nu_{2}$, which is nothing but $\sin ^{2} \theta$, without anomalous seasonal variations. Therefore, the sensitivity to the anomalous seasonal variation is reduced in the region with small $P_{c}$. When $\Delta m^{2}$ is small, on the other hand, the situation is in the extreme non-adiabatic limit, and $P_{c} \rightarrow \cos ^{2} \theta$. Then Eq. (3.8) reduces to Eq. (3.1). As $\Delta m^{2}$ increases, $P_{c}$ becomes smaller than $\cos ^{2} \theta$, which enhances the vacuum oscillation effect in the small mixing angle region. Curve 2 in Fig. 7 includes the matter effect and indeed indicates a reduced sensitivity for large $\sin ^{2} 2 \theta\left(\right.$ small $P_{c}$ ) and an enhanced sensitivity for small $\sin ^{2} 2 \theta$ (where $P_{c}$ starts deviating from $\cos ^{2} \theta$ ).

The second effect is the finite width of the ${ }^{7} \mathrm{Be}$ line. To give some preliminary idea about the relative size of this effect, we first consider a simplified model. We assume for a moment that the only source of the line broadening is the Doppler shift of neutrino energies arising from the thermal motion of the ${ }^{7}$ Be nuclei. Since the energy is shifted to $E \rightarrow E\left(1+v_{z} / c\right)$ and the probability distribution of the velocity along the line of sight $v_{z}$ is proportional to $\exp \left(-m v_{z}^{2} / 2 k T\right)$, the resulting line profile will be a Gaussian $\exp \left(-m c^{2}\left(E-E_{0}\right)^{2} /\left(2 k T E_{0}^{2}\right)\right)$. Taking the temperature to be 15.6 million Kelvin (the temperature in the center of the Sun) and integrating over the line profile, we obtain curve 3 in Fig. 7. The sensitivity loss now occurs at $\Delta m^{2} \simeq 1 \times 10^{-8} \mathrm{eV}^{2}$, demonstrating that this effect is more important than the matter effect.

This naive model is actually incomplete; there exists another very important source of line broadening. Because the incoming electron in the process ${ }^{7} \mathrm{Be}+e^{-} \rightarrow{ }^{7} \mathrm{Li}+\nu_{e}$ has nonzero thermal kinetic energy, the center of mass energy of the reaction is greater than the one measured in the laboratory, and so the neutrino has a greater energy. The phase space distribution of electrons is governed by the Maxwellian factor $\exp \left(-E_{e^{-}} / k T\right)$. This distribution has to be multiplied by the energy-dependent cross section, integrated over the phase space, and finally convoluted with the Gaussian arising from the Doppler effect. The resulting line shape becomes asymmetric, with a Gaus-

\footnotetext{
"In the numerical scan, we ignored the additional phase factor $\delta$, because its effects are negligible [24].
} 
sian profile on the left (due to the Doppler effect) and an exponential tail on the right (due to the Maxwellian distribution of the electron energy). The issue was studied in detail in [27], where the precise form of the profile was computed. ${ }^{* *}$ Repeating the calculation with this profile we generate curve 4 in Fig. 7 .

One can see that for this curve the cut-off occurs at smaller $\Delta m^{2}$. This behavior is expected, because the linewidth is now greater than when only the Doppler effect was included (curve 3 in Fig. 7). It is also worth noting that the cut-off sets in more gradually. This feature can be understood analytically by considering the Fourier transform of an exponential tail vs. a Gaussian tail. The details can be found in Appendix B.

Finally, we can combine both the linewidth and the matter effects. The result is curve 5 in Fig. 7. As expected, the inclusion of the matter effect on top of the linewidth effect introduces only a small distortion to the sensitivity region. It is important to note that for $\Delta m^{2} \lesssim 5 \times 10^{-10} \mathrm{eV}^{2}$ none of the physical effects mentioned above affect the sensitivity region (curve 1 versus curve 5, in Fig. 7).

We need to consider one last ingredient in the analysis. We again return to the issue of the number of bins. While choosing more bins is necessary for larger values of $\Delta m^{2}$, it simultaneously leads to a loss of sensitivity for smaller $\Delta m^{2}$. A better procedure is to use an optimum number of bins $N_{\text {opt }}$ for each $\Delta m^{2}$. It can be shown that for our method of analysis (minimizing $\chi^{2}$ by varying the signal and background) and sufficiently large $\Delta m^{2}$ an approximate formula holds: $N_{\text {opt }} \simeq 2 \times 10^{10}\left(\Delta m^{2} / 1 \mathrm{eV}^{2}\right)$. Of course, this formula should not be used when the optimal number of bins it predicts is too small. We choose to use 12 bins for $\Delta m^{2} \leq 6 \times 10^{-10} \mathrm{eV}^{2}$ and a variable number of bins $N_{\text {bins }}=2 \times 10^{10}\left(\Delta m^{2} / 1 \mathrm{eV}^{2}\right)$ for $\Delta m^{2}>6 \times 10^{-10} \mathrm{eV}^{2}$.

In Fig. 9 we show the entire sensitivity reach of Borexino after three years of running. The unshaded region will be covered at least at 95\% CL, if in the analysis one allows the background and the incoming solar neutrino flux to float. The dark shading marks the additional portion of the parameter space

**It turns out that other effects, such as collisional line broadening [28] or gravitational energy shift [27], are unimportant.

${ }^{\dagger}$ An alternative technique, which can be considered more rigorous but which would also be more computer intensive, is to Fourier transform the simulated data for every value of $\left(\sin ^{2} 2 \theta, \Delta m^{2}\right)$ in the scan. One can then compare the intensities of the harmonics to those expected for the case of no oscillations. A description of this method can be found in 29]. For our purposes varying the number of bins is sufficient. 
that will be covered at least at 95\% CL, if in the analysis one assumes both the anticipated background rate (10\% uncertainty) and the SSM prediction of the ${ }^{7}$ Be solar neutrino flux (9\% uncertainty). For $\Delta m^{2} \gtrsim 5 \times 10^{-9} \mathrm{eV}^{2}$, the sensitivity to the anomalous seasonal variation gets lost because of the smearing due to the linewidth effect. However, there is an overall suppression of the flux due to the MSW effect in this region. To be sensitive to this overall suppression, we should return to a smaller number of bins to enhance the statistical accuracy. We therefore use 12 bins in this region.

For comparison, we also superimpose the "just-so" preferred regions obtained by analyzing the total event rates in the Homestake, GALLEX, SAGE, and Super-Kamiokande experiments (Fig. 5 in [0]). The plot shows that Borexino will be sensitive to almost all of the preferred region, even without relying on the SSM prediction of the incoming neutrino flux or on the knowledge of the background rate. Only two thin spikes protrude through the lower "islands". This overlap disappears completely when the anticipated background rate and the SSM prediction for the incoming neutrino flux are used in the "data" analysis, in which case the entire preferred region is covered.

Fig. 10 contains a similar plot for three years of KamLAND running. Because KamLAND will have more statistics, it will be sensitive at 95\% CL to the entire preferred region without relying in the analysis on the SSM prediction of the incoming neutrino flux or on the knowledge of the background rate.

As mentioned earlier, the sensitivity to anomalous seasonal variations is completely lost for $\Delta m^{2} \gtrsim 10^{-8} \mathrm{eV}^{2}$. In this case the seasonal variation of the data is consistent with an average suppression of the incoming neutrino flux. In particular, in the case of the MSW solutions $\left(10^{-7} \mathrm{eV}^{2} \lesssim \Delta m^{2} \lesssim\right.$ $10^{-4} \mathrm{eV}^{2}$ ), no anomalous seasonal variations can be detected, as was implicitly assumed in Sec. 2 .

At last, it is worth mentioning that the experiments will still be sensitive to a significant part of the preferred region even if the background rate or the incoming ${ }^{7} \mathrm{Be}$ neutrino flux (for all flavors) turns out to be significantly different. For example, if the background rate at Borexino (KamLAND) turns out to be 30 (100) times higher than expected, the part of the preferred

\footnotetext{
${ }_{\ddagger}^{\ddagger}$ One can cover a slightly larger portion of the parameter space by using yet fewer bins. We chose 12 bins such that one can still verify the expected $1 / L^{2}$ behavior of the signal even with a reduced flux, as we discussed in Sec. 2 .
} 


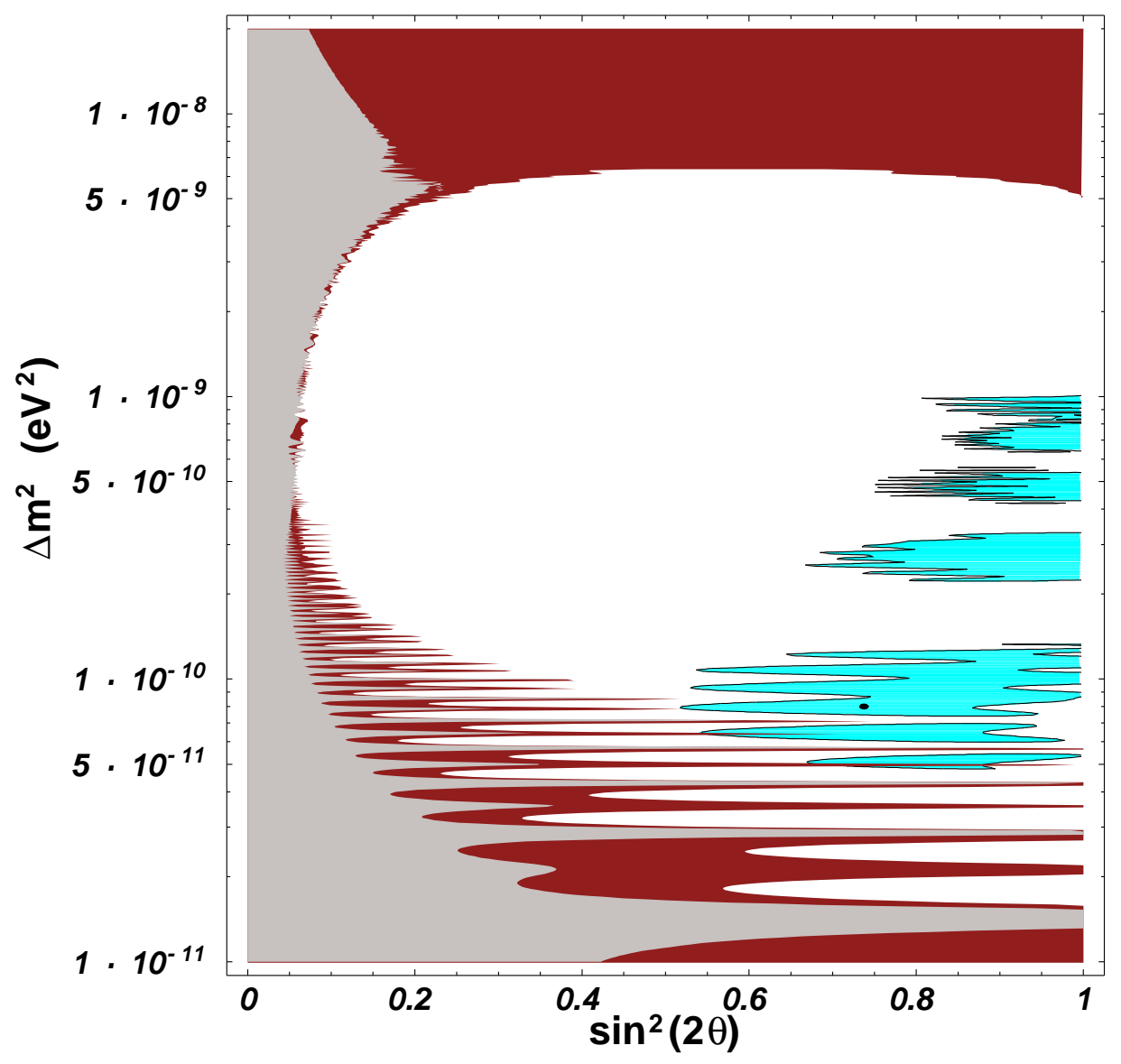

Figure 9: The final sensitivity plot for three years of Borexino running, after the inclusion of all effects limiting the reach of the experiment for large $\Delta m^{2}$. The white region corresponds to the sensitivity at more than $95 \%$ confidence level with both the incoming neutrino flux and background rate assumed to be unknown, and the dark region to the additional coverage when the SSM ${ }^{7} \mathrm{Be}$ flux and the background rate estimated elsewhere are used. Also shown are the regions preferred by the analysis of the total rates in the Homestake, GALLEX, SAGE, and Super-Kamiokande experiments [7]. 


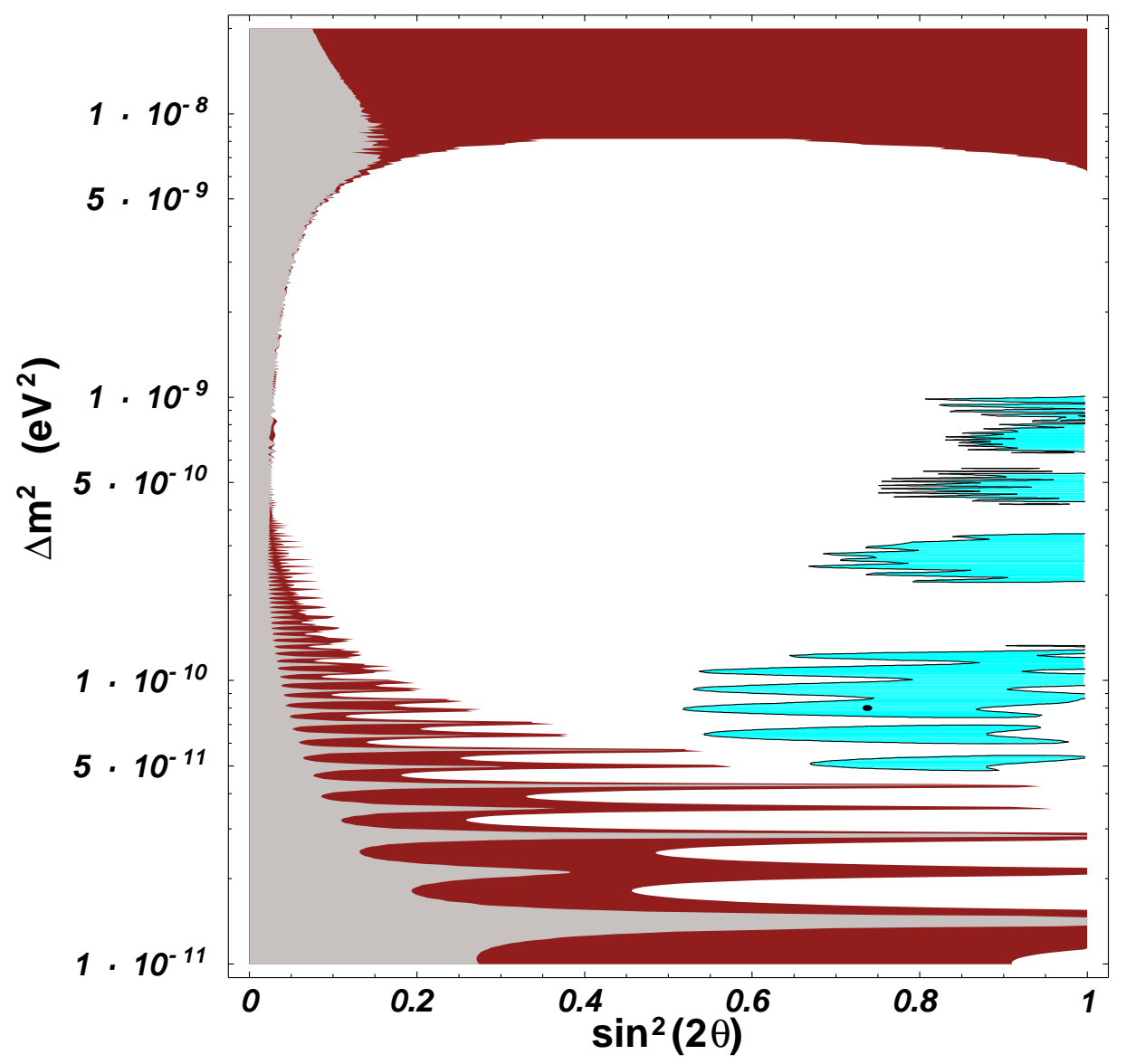

Figure 10: The same as Fig 9, but for three years of KamLAND running. 
region with $\Delta m^{2}>10^{-10} \mathrm{eV}^{2}$ will still be within the reach of the experiment, after three years of running. The sensitivity will be completely lost only if the background rate turns out to be three (four) orders of magnitude higher than anticipated at Borexino (KamLAND). The consequences of a ${ }^{7}$ Be solar neutrino flux smaller than predicted by the SSM can also be studied. If the ${ }^{7}$ Be neutrino flux is for some reason suppressed by a factor of 5 , KamLAND is still sensitive to the part of the preferred region with $\Delta m^{2}>10^{-10} \mathrm{eV}^{2}$, after 3 years of running.

\section{Measuring the Oscillation Parameters}

In this section, we address the issue of how well the two-neutrino oscillation parameters, $\sin ^{2} 2 \theta$ and $\Delta m^{2}$, can be extracted if the data collected at future solar neutrino experiments exhibits an anomalous seasonal variation. In order to do this, we simulate "data", according to the procedure developed in Sec. 3, for two distinct points in the parameter space, $\sin ^{2} 2 \theta=0.7, \Delta m^{2}=$ $8 \times 10^{-11} \mathrm{eV}^{2}$ ("low point") and $\sin ^{2} 2 \theta=0.9, \Delta m^{2}=4.5 \times 10^{-10} \mathrm{eV}^{2}$ ("high point"). The low point is close to the best fit point presented in [7], while the high point is close to the point preferred by the Super-Kamiokande analysis of the recoil electron energy spectrum [30. The data is binned into months (12 bins per years), and Fig. 11 depicts the annual variations for both the high and the low points, assuming three years of Borexino running. The no-oscillation case is also shown.

In order to measure the oscillation parameters, we perform a 4 parameter $\left(s, b, \sin ^{2} 2 \theta\right.$, and $\left.\Delta m^{2}\right)$ fit to the "data". The fit is performed by minimizing $\chi^{2}$ with respect to the incoming neutrino flux $(s)$ and the background rate (b), as in Sec. 3, and computing it for fixed $\sin ^{2} 2 \theta$ and $\Delta m^{2}$. Fig. 12 depicts the values of $\left(\sin ^{2} 2 \theta, \Delta m^{2}\right)$ and the $95 \%$ CL contours (for two degrees of freedom), extracted from the "data" consistent with the low (light) and high (dark) points. Note that this is very different from what was done in the previous section. There, for each point in the $\left(\sin ^{2} 2 \theta, \Delta m^{2}\right)$ plane there was a different "data" set, and the "data" was fitted by a non-oscillation theoretical function. Here the "data" is fixed (either the low or the high point), and is fitted by a theoretical function which assumes neutrino oscillations.

One should easily note that the extracted 95\% CL contour for the high point consists of only two "islands", while for the low point one extracts a collection of "islands". The reason for this is simple. When $\Delta m^{2} \sim$ few $\times$ 


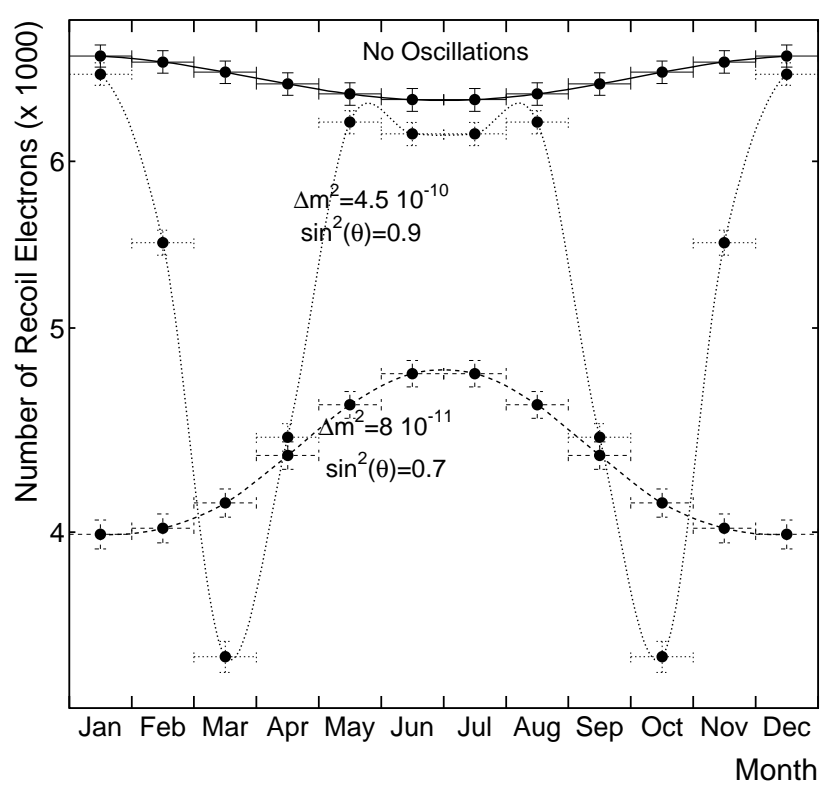

Figure 11: Number of recoil electrons detected in a given month, for the low point, the high point (see text for description) and the case of no neutrino oscillations, after three years of Borexino running.

$10^{-10} \mathrm{eV}^{2}$, the oscillation length is slightly smaller than $\Delta L$ (see Eq. (2.5)). This means that the seasonal variation of the "data" has a very particular shape (as one may easily confirm by looking at Figs. 1, 11), which cannot be easily mimicked by other values of $\Delta m^{2}$, even when the background rate and the incoming flux are varied in the fit procedure.

When $\Delta m^{2} \sim$ several $\times 10^{-11} \mathrm{eV}^{2}$, the oscillation length is larger than $\Delta L$, and the effect of seasonal variations is less pronounced. There is a collection of $\Delta m^{2}$ 's that yields the same qualitative behavior. Because our fit procedure allows for the background rate and the neutrino flux to float freely, a good agreement with the "data" is met for a large portion of the parameter space. In order to make this discussion clearer, it is useful to describe in detail what happens to the number of electron neutrinos reaching the detector as a function of time.

In the case of the low point: initially, when the Earth is at the perihelion, the $\nu_{e}$ survival probability is small and, as time progresses, monotonically increases until the Earth reaches the aphelion (after six months). The pro- 


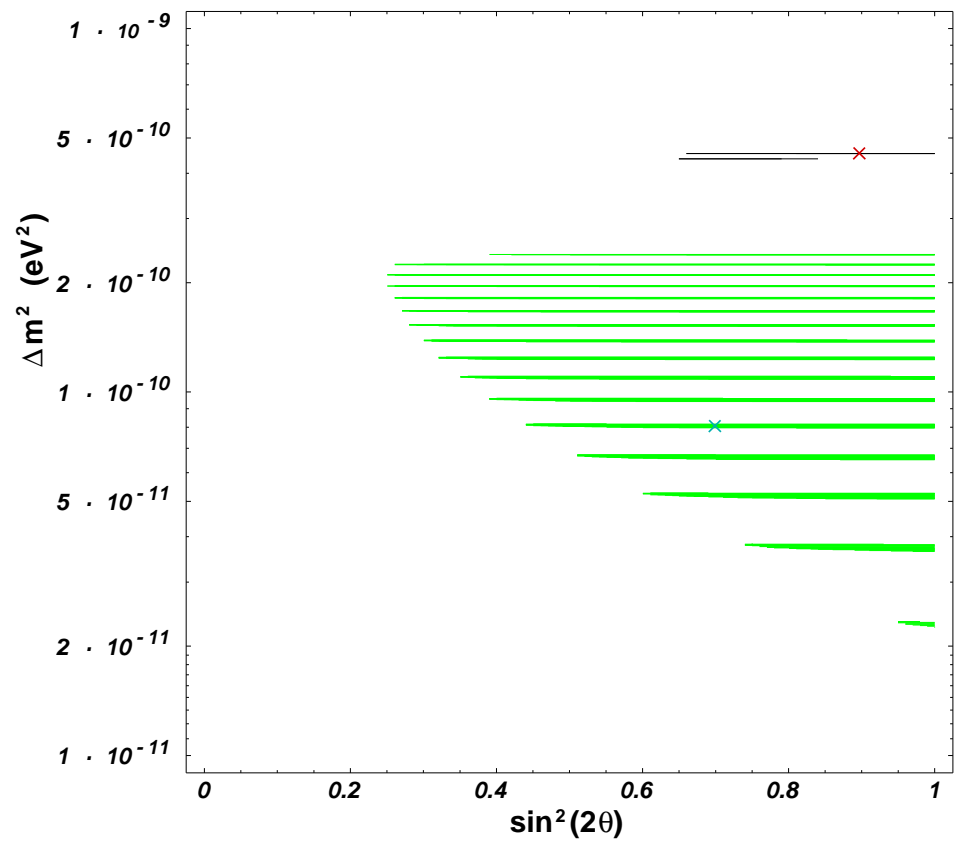

Figure 12: Measurement of the neutrino oscillations parameters $\sin ^{2} 2 \theta$ and $\Delta m^{2}$, assuming no knowledge of the SSM and the number of background events. The regions represent the $95 \%$ confidence level contours, for data consistent with the high (dark) and low points (light). The input points are indicated in the figure by the two crosses. See text for details. We assume 3 years of Borexino running.

cess happens in reverse order in the next six months, as expected. There are many other values of the oscillation length, i.e. $\Delta m^{2}$, such that the survival probability monotonically increases for increasing Earth-Sun distance and therefore a similar qualitative behavior is to be expected. The main quantitative difference is in the ratio of the number of events detected in the perihelion and in the aphelion, which may be accounted for by varying the background rate and the incoming neutrino flux. This explains the existence of islands. For values of $\Delta m^{2}$ in between islands, the survival probability either increases and decreases for varying Earth-Sun distance, or monotonically decreases. The exact location of the islands and their widths can only be understood by analyzing the fit procedure, in particular the minimization of $\chi^{2}$ with respect to the background rate and the incoming neutrino flux. Note that there are no "islands" above $\Delta m^{2} \gtrsim 2.5 \times 10^{-10} \mathrm{eV}^{2}$. This is be- 
cause when the oscillation length is small enough (or $\Delta m^{2}$ large enough), the survival probability cannot only increase for increasing Earth-Sun distance, but necessarily reaches a maximum before the aphelion, and then decreases, independent of what the survival probability at the perihelion is. This situation is qualitatively different from the low point.

In the case of the high point: initially the survival probability is close to unity, decreases sharply as the Earth moves further from the Sun, and then grows rapidly, reaching a maximum when the Earth is close to its aphelion, because the oscillation length is smaller than $\Delta L$. In this case, little variations in the oscillation length, i.e. $\Delta m^{2}$, produce big qualitative changes, including the position and number of maxima and minima. There is still a small ambiguity (i.e. two "islands") in determining $\Delta m^{2}$ for the high point. This happens when the oscillation length is such that the minimum of the survival probability happens in March/October and the survival probability is large enough at the perihelion and the aphelion. The fact that the absolute values of the number of recoil electrons detected are different is taken care of by varying the signal and the background.

In conclusion, if Nature chose neutrino oscillation parameters such that $\sin ^{2} 2 \theta$ is large and $\Delta m^{2} \approx$ few $\times 10^{-10} \mathrm{eV}^{2}$, Borexino should be able to measure these parameters independent of the SSM and any knowledge of the number of background events, with good precision (especially in $\Delta m^{2}$ ). If $\Delta m^{2} \approx$ several $\times 10^{-11} \mathrm{eV}^{2}$, the determination of oscillation parameters is not as precise. Better precision can be achieved at KamLAND, but the ambiguity of solutions in the "low" $\Delta m^{2}$ region still remains.

\section{Exclusion of Vacuum Oscillations}

In this section, we address the issue of what the experiments can conclude about vacuum oscillations if no discrepancy from the normal seasonal variation effect is detected. In this case, one may be able to measure the incoming neutrino flux, as outlined in Sec. 2. Two distinct possibilities will be considered: (1) the measured flux is consistent with the SSM prediction; (2) the measured flux is suppressed with respect to the SSM prediction.

In the first case, one would be inclined to trust the SSM prediction of the ${ }^{7}$ Be neutrino flux and use it in the analysis to exclude vacuum oscillations. This will be discussed in Sec. 5.1. On the other hand, in the second case, it is not clear if the reduced flux is due to MSW neutrino oscillations, an 
incorrect SSM prediction of the neutrino flux, etc. This will be discussed in Sec. 5.2.

\subsection{If the Flux is Consistent with the SSM Prediction}

We simulate "data" consistent with the SSM and the expected number of background events. The relevant numbers are quoted in Sec. 2. The "data" are binned into months (12 bins per year), and are illustrated in Fig. 11, assuming three years of Borexino running. We then fit to the "data" annual distributions that include neutrino oscillations for a given choice of $\left(\sin ^{2} 2 \theta, \Delta m^{2}\right)$, plus a constant background. The background rate and the incoming neutrino flux may be allowed to float in the fit, constrained to a positive number.

It is important to note that this is the opposite of what was done in Sec. 3, where the sensitivity of Borexino and KamLAND to vacuum oscillations was studied. There, the simulated "data" were consistent with vacuum oscillations, and one tried to fit a non-oscillation prediction to the "data" by varying the incoming flux and/or the background. Here, the "data" are consistent with no oscillations, and one tries to fit the "data" with a prediction which includes the effect of neutrino oscillations for fixed $\left(\sin ^{2} 2 \theta, \Delta m^{2}\right)$, by varying the incoming flux and/or the background. If both the background and the incoming flux are fixed, i.e. not allowed to vary in the fit procedure, the exclusion and the sensitivity regions are the same. On the other hand, if both the background rate and the incoming flux are allowed to float, the exclusion region is expected to be smaller than the sensitivity region presented in Sec. 3, especially in the region $\Delta m^{2} \lesssim 10^{-10} \mathrm{eV}^{2}$. This is due to the fact that a large number of points in the parameter space yield an annual variation of the $\nu_{e}$ flux which is much larger than $7 \%$, but agrees with the shape of the normal seasonal variation. If in the fit procedure the signal is scaled down to reduce the amplitude of the variation and the background scaled up to increase the number of events, a good fit to the no oscillation case can be attained.

Fig. 13 shows, for three years of Borexino and KamLAND running, the region of the $\left(\sin ^{2} 2 \theta, \Delta m^{2}\right)$ parameter space excluded at $95 \% \mathrm{CL}$, if one allows the solar neutrino flux and the background rate to float within the positive numbers (in white), and if one assumes the solar neutrino flux calculated in the SSM within theoretical errors (in light plus white).

A few comments are in order. First, one notices that the KamLAND 

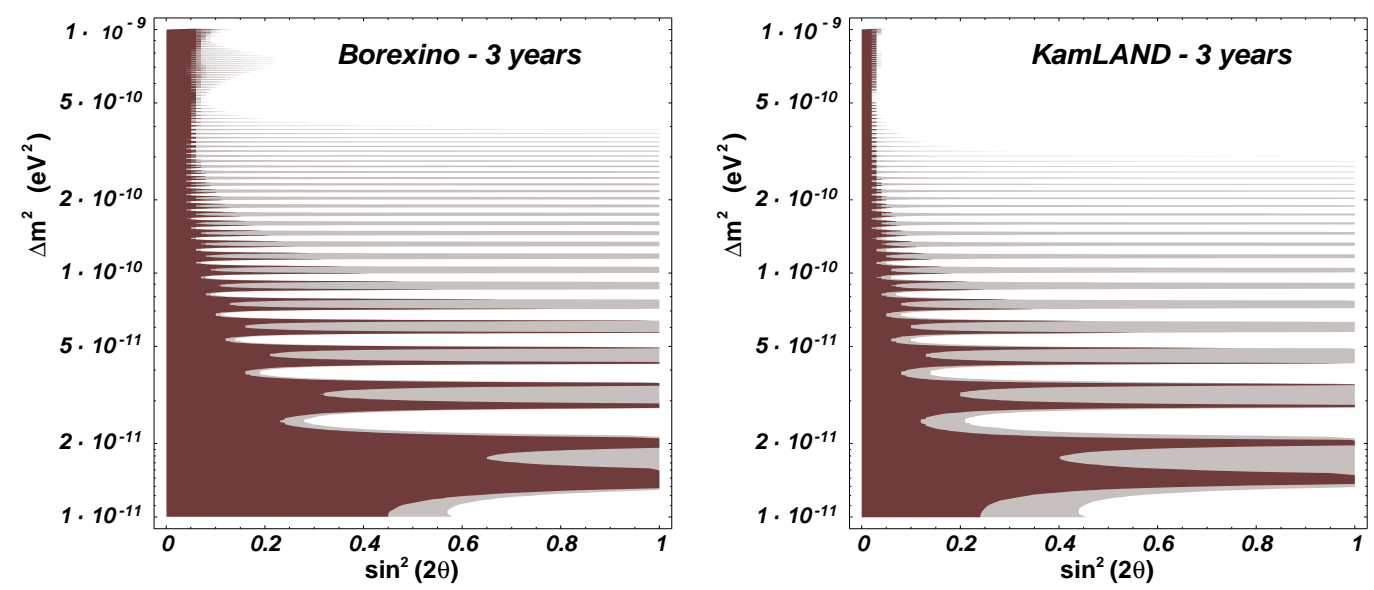

Figure 13: Region of the two neutrino oscillation parameter space excluded in the case of no neutrino oscillations if one assumes no knowledge of the background and no knowledge of the SSM (white) or knowledge of the SSM (light+white), after 3 years of Borexino (right) and KamLAND (left) running.

exclusion region is larger than the one excluded by Borexino. This is, of course, expected because of KamLAND's larger fiducial volume and therefore higher statistics. Second, when the solar neutrino flux is allowed to vary in the fit, the excluded region of the parameter space shrinks, as expected and discussed earlier. Third, one can safely claim that, if no discrepancies are detected in the seasonal variation spectrum, the "large" $\Delta m^{2}$ (several $\times 10^{-10} \mathrm{eV}^{2}$ ) set of vacuum solutions (see Figs. 9 and 10) will be excluded, even at Borexino. Even when no knowledge of the incoming neutrino flux is used, a reasonable portion of the "small" $\Delta m^{2}\left(\right.$ several $\left.\times 10^{-11} \mathrm{eV}^{2}\right)$ set of solutions is also excluded. When one assumes knowledge of the incoming neutrino flux, the entire allowed region is excluded.

If the background rate is larger than expected, the excluded region diminishes accordingly. This is because when the constant background is enhanced with respect to the oscillation signal it is easier to achieve a reasonable $\chi^{2}$ for the fit even when the seasonal variations due to vacuum oscillations are significantly different from the no-oscillation case. In particular, when the background rate is large enough that the seasonal distribution of the data is statistically consistent with a flat one, a reasonable $\chi^{2}$ for the fit can always be achieved simply by scaling the signal to zero and scaling up the back- 
ground appropriately. Explicitly, after three years of Borexino (KamLAND) running the exclusion region vanishes if the background rate is $\sim 8$ (40) times larger than anticipated, when both the background rate and the incoming neutrino flux are allowed to float in the fit or $~ 500$ (3000) times larger than anticipated when one assumes the neutrino flux predicted by the SSM.

\subsection{If There is an Overall Suppression of the Flux}

If there is an overall, i.e., time-independent suppression of the flux (which is the case for the MSW solutions), the way to proceed towards excluding part of the vacuum oscillation parameter space is less clear. This is because such an experimental result neither agrees with the SSM prediction nor does it represent any "smoking gun" signature for neutrino oscillations, as is the case of anomalous seasonal variations. One does not know if the SSM prediction of the flux is simply wrong, or if there are neutrino oscillations consistent with one of the MSW solutions or both. Anyway, it is clear that (in general) the incoming neutrino flux should be considered unknown in the data analysis.

The most conservative option is to follow the same analysis done in the previous subsection, and allow both the incoming neutrino flux and the background rate to float in the fit. In this case, the excluded region of the two-neutrino oscillation parameter space is reduced significantly, and may completely disappear. This is because when the number of signal events is reduced the annual distribution is closer to flat and a good fit is obtained even when the would-be annual variations are very different. This is very similar to what was previously discussed at the end of the last subsection, where we discussed what happens if the background rate turns out to be much larger than anticipated. Explicitly, after three years of Borexino running and a signal rate which is $21.3 \%$ of the SSM prediction (as one would obtain in the case of the small angle MSW solution), Borexino is unable to exclude any portion of the vacuum oscillation parameter space, while KamLAND can still exclude about one half of the "high" and "low" $\Delta m^{2}$ preferred regions. If the background rate can be estimated by other means with $10 \%$ uncertainty, Borexino and KamLAND will be able to exclude the entire "high" $\Delta m^{2}$ region and a significant portion of the "low" $\Delta m^{2}$ region.

In order to go beyond the most conservative analysis discussed above, one would have to look at the overall situation of the solar neutrino puzzle at the time of the data analysis. It is likely that one will be able to do much better. For example, solar neutrino oscillations might have already 
been established by the SNO experiment [31], and perhaps it is reasonable to assume the incoming solar neutrino flux predicted by the SSM. Then it would be possible to exclude a region of the parameter space as large as the one in Sec. 5.1 where one assumes the SSM flux. Another possibility is that Super-Kamiokande or SNO rules out the small angle MSW solution by studying the distortions of the electron energy spectrum [30, 31], and a large suppression of the ${ }^{7} \mathrm{Be}$ solar neutrino flux would indicate that there is something wrong with the SSM. In this case, it is not clear how to proceed. We do not go into further discussions on all logical possibilities.

\section{Conclusions}

We have studied possible uses of the seasonal variation of the ${ }^{7}$ Be solar neutrino flux at Borexino and KamLAND. Our results can be summarized as follows. Once the experiments accumulate enough data to see seasonal variations, the first step will be to determine if the observed pattern is consistent with the normal $1 / L^{2}$ flux suppression. If a discrepancy is found, it will be a sign of vacuum oscillations. In this case, the seasonal variation of the data can be used to determine the oscillation parameters $\sin ^{2} 2 \theta$ and $\Delta m^{2}$. On the other hand, if the data are consistent with the normal pattern, the amplitude of the variation can be used to measure the ${ }^{7}$ Be solar neutrino flux and to exclude a significant portion of the vacuum oscillation parameter space.

If the observed seasonal variations are consistent with the normal $1 / L^{2}$ flux suppression, one can use the amplitude of the variation to determine what fraction of the observed recoil electrons are induced by the neutrinos coming from the Sun. This method is limited by statistics, and the accuracy is worse when the ${ }^{7} \mathrm{Be}$ solar neutrino flux is suppressed, as in the case of the small angle MSW solution. In fact, in Sec. 2 we found that in the case of a large suppression only KamLAND should be able to perform such a measurement, after 3 years of data taking. It is important to emphasize that we assumed the oscillation of electron neutrinos into other active flavors. In the case of oscillations into sterile neutrinos, the ${ }^{7} \mathrm{Be}$ solar neutrino flux might be almost absent, and in this case neither Borexino nor KamLAND are able to perform a measurement of the flux using this technique.

An important advantage of this technique is that it does not require a separate estimate of the background rate, which may be a very difficult task. If the background rate can be reliably measured by some other means, one 
can obtain another measurement of the neutrino flux. In this case, the two results can then be compared for consistency, thus making the final result on the ${ }^{7}$ Be neutrino flux much more trustworthy.

We also studied in great detail the effect of vacuum neutrino oscillations on seasonal variations. Our analysis shows that the outlook for discovering vacuum oscillations at both Borexino and KamLAND is very favorable. A very important finding in Sec. 3 is that the experiments may detect a deviation from the normal pattern of seasonal variations even without relying on the SSM prediction of the incoming neutrino flux or estimate of the background rate. The analysis would consist of trying to fit the observed data with the normal $1 / L^{2}$ pattern, treating the incoming neutrino flux and the background rate as free parameters. With this technique, after three years of running Borexino should detect anomalous seasonal variations for almost all values of $\left(\sin ^{2} 2 \theta, \Delta m^{2}\right)$ preferred by the analysis of the neutrino flux data from Homestake, GALLEX, SAGE, and Super-Kamiokande, as illustrated in Fig. 9. The sensitivity region should be larger at KamLAND (Fig. 10). Results obtained in this way would be very robust. Both experiments are sensitive to an even larger portion of the parameter space if the background rate can be reliably estimated by auxiliary measurements.

If anomalous seasonal variations are discovered, the data can be used to measure the oscillation parameters $\left(\sin ^{2} 2 \theta, \Delta m^{2}\right)$. This issue was studied in Sec. 4. It was found that for $\Delta m^{2} \gtrsim 10^{-10} \mathrm{eV}^{2}$ the experiments will be able to determine $\Delta m^{2}$ with good precision. At the same time, for $\Delta m^{2} \lesssim 10^{-10} \mathrm{eV}^{2}$ there would be many "candidate islands" in the $\left(\sin ^{2} 2 \theta, \Delta m^{2}\right)$ plane, and it will not be easy to resolve the ambiguity.

On the other hand, the absence of anomalous seasonal variations of the ${ }^{7}$ Be solar neutrino flux data can be used to exclude regions of the vacuum oscillation parameter space. In Sec. 5 we presented the exclusion plots for both Borexino and KamLAND, after three years of running. An important lesson from that section is that in order to exclude a large portion of the preferred region, the experiments will need to either measure the background rate or rely on the SSM prediction for the neutrino flux. In the absence of both, the results are rather weak. This is to be contrasted with the situation in Sec. 3 .

It is important to keep in mind that the simulated "data" is most of the time based on the SSM prediction for the ${ }^{7} \mathrm{Be}$ solar neutrino flux and the anticipated number of background events at Borexino and KamLAND. Our numerical results, therefore, even in the cases when we do not use the knowl- 
edge of the incoming neutrino flux or the background rate at the analysis stage, are not to be regarded as SSM and background rate independent. We would like to draw attention to our comments at the end of Secs. 2 and 3 on how our results might change if these inputs are changed. We also assume only statistical errors in the data analysis, neglecting systematic uncertainties due to the lack of knowledge in the seasonal variation of the background rate. The inclusion of such effects is beyond the scope of this paper.

Overall our results indicate that the future Borexino results can lead to significant progress towards solving the solar neutrino puzzle. Furthermore, if KamLAND is also able to study solar neutrinos, one would have access to a larger data set, and more powerful results can be obtained.

\section{Acknowledgments}

We would like to express special thanks to Eligio Lisi and Lincoln Wolfenstein who pointed out the irrelevance of the core size effect. HM also thanks John Bahcall, Stuart Freedman, and Kevin Lesko for useful conversations. This work was supported in part by the U.S. Department of Energy under Contracts DE-AC03-76SF00098, in part by the National Science Foundation under grant PHY-95-14797. HM was also supported by Alfred P. Sloan Foundation, and AdG by CNPq (Brazil).

\section{A $\chi^{2}$ Analysis}

In the analyses in Sec. 3, 4, and 5, we are interested in the capability of an "average" experiment. It is possible to simulate "data" with statistical

fluctuations included, but then the value of $\chi^{2}$ would vary slightly between different repetitions of the same simulation. A better approach is to find an expression for $\chi^{2}$ "averaged" over many simulations. As we show below, averaging over statistical fluctuations simply leads to the inclusion of a constant term in the definition of $\chi^{2}$.

Suppose we have some solar neutrino data binned into $N_{\text {bins }}$ bins. Let the average expected value in the $i$ th bin be $d_{i}$ with corresponding random fluctuation $\Delta d_{i}$. Suppose we want to fit this data with a function $f$, which can depend on two parameters: the signal $s$ and the background $b$. Then the 
$\chi^{2}$ of the fit can be defined as follows:

$$
\chi^{2}(s, b)=\sum_{i}^{N_{\text {bins }}} \frac{\left(d_{i}+\Delta d_{i}-f_{i}(s, b)\right)^{2}}{\sigma_{d_{i}}^{2}},
$$

where $\sigma_{d_{i}}=\sqrt{d_{i}+\Delta d_{i}}$. Because, in the case of interest, the number of events per bin is sufficiently large, we can approximately set $\sigma_{d_{i}} \simeq \sqrt{d_{i}}$. .

First consider the case when $s$ and $b$ are fixed numbers. The average value of the $\chi^{2}$ one would obtain after simulating the data many times is

$$
\left\langle\chi^{2}\right\rangle=\left\langle\sum_{i}^{N_{\text {bins }}}\left[\frac{\left(\Delta d_{i}\right)^{2}}{d_{i}}+\frac{2 \Delta d_{i}\left(d_{i}-f_{i}\right)}{d_{i}}+\frac{\left(d_{i}-f_{i}\right)^{2}}{d_{i}}\right]\right\rangle .
$$

Using $\left\langle\Delta d_{i}\right\rangle=0,\left\langle\left(\Delta d_{i}\right)^{2}\right\rangle=d_{i}$, we find

$$
\left\langle\chi^{2}\right\rangle=\sum_{i}^{N_{\text {bins }}}\left[1+\frac{\left(d_{i}-f_{i}\right)^{2}}{d_{i}}\right]=N_{\text {bins }}+\sum_{i}^{N_{\text {bins }}} \frac{\left(d_{i}-f_{i}\right)^{2}}{d_{i}} .
$$

Therefore, in this simplest case it is enough to use the average values $d_{i}$ and the number of bins to compute $\left\langle\chi^{2}\right\rangle$.

Next, consider the case when $f(s, b)=b+g(s)$ and $\chi^{2}$ is minimized with respect to $b$.

$$
\begin{aligned}
\frac{\partial \chi^{2}(s, b)}{\partial b} & =-\sum_{i}^{N_{\text {bins }}} \frac{2\left(d_{i}+\Delta d_{i}-b-g_{i}(s)\right)}{d_{i}}=0 \\
\Longrightarrow b & =\left(\sum_{i}^{N_{\text {bins }}} \frac{\left(d_{i}+\Delta d_{i}-g_{i}(s)\right)}{d_{i}}\right)\left(\sum_{i}^{N_{\text {bins }}} \frac{1}{d_{i}}\right)^{-1} .
\end{aligned}
$$

Introducing $A_{i} \equiv\left(d_{i}+\Delta d_{i}-g_{i}(s)\right) / d_{i}$ and substituting Eq. (A.4) in Eq. (A.1), we obtain

$$
\begin{aligned}
\chi_{\text {min }}^{2} & =\sum_{i}^{N_{\text {bins }}}\left[\frac{A_{i}^{2}}{d_{i}}-2 \frac{A_{i}}{d_{i}}\left(\sum_{i}^{N_{\text {bins }}} \frac{A_{i}}{d_{i}}\right)\left(\sum_{i}^{N_{\text {bins }}} \frac{1}{d_{i}}\right)^{-1}+\frac{1}{d_{i}}\left(\sum_{i}^{N_{\text {bins }}} \frac{A_{i}}{d_{i}}\right)^{2}\left(\sum_{i}^{N_{\text {bins }}} \frac{1}{d_{i}}\right)^{-2}\right] \\
& =\sum_{i}^{N_{\text {bins }}} \frac{A_{i}^{2}}{d_{i}}-\left(\sum_{i}^{N_{\text {bins }}} \frac{A_{i}}{d_{i}}\right)^{2}\left(\sum_{i}^{N_{\text {bins }}} \frac{1}{d_{i}}\right)^{-1} .
\end{aligned}
$$

* One can easily estimate the resulting relative error in $\chi^{2}$ to be of $O\left(1 / \sqrt{\left\langle d_{i}\right\rangle}\right)$. 
Now plugging back in the definition of $A_{i}$, we perform the averaging using $\left\langle\Delta d_{i}\right\rangle=0,\left\langle\left(\Delta d_{i}\right)^{2}\right\rangle=d_{i}$, and $\left\langle\left(\Delta d_{i}\right)\left(\Delta d_{j}\right)\right\rangle=0$ for $i \neq j$ :

$$
\begin{aligned}
\left\langle\sum_{i}^{N_{\text {bins }}} \frac{A_{i}^{2}}{d_{i}}\right\rangle & =N_{\text {bins }}+\sum_{i}^{N_{\text {bins }}} \frac{\left(d_{i}-f_{i}\right)^{2}}{d_{i}} \\
\left\langle\left(\sum_{i}^{N_{\text {bins }}} \frac{A_{i}}{d_{i}}\right)^{2}\right\rangle & =\left(\sum_{i}^{N_{\text {bins }}} \frac{1}{d_{i}}\right)+\left(\sum_{i}^{N_{\text {bins }}} \frac{\left(d_{i}-g_{i}(s)\right)}{d_{i}}\right)^{2} .
\end{aligned}
$$

Substituting Eq. (A.6) in Eq. (A.5), we find

$$
\left\langle\chi_{\min }^{2}\right\rangle=N_{\text {bins }}-1+\sum_{i}^{N_{\text {bins }}} \frac{\left(d_{i}-f_{i}\right)^{2}}{d_{i}}-\left(\sum_{i}^{N_{\text {bins }}} \frac{\left(d_{i}-g_{i}(s)\right)}{d_{i}}\right)^{2}\left(\sum_{i}^{N_{\text {bins }}} \frac{1}{d_{i}}\right)^{-1} .
$$

The last two terms are exactly what one would find after minimizing $\sum_{i}^{N_{\text {bins }}}\left(d_{i}-\right.$ $\left.b-g_{i}(s)\right)^{2} / d_{i}$ with respect to $b$, and hence in this case random fluctuations can be accounted for by replacing $N_{\text {bins }}$ in Eq. (A.3) by $N_{\text {bins }}-1$.

One can easily show that, if $f(s, b)_{i}=b+s \cdot h_{i}$ and one minimizes $\chi^{2}$ with respect to $s$, the effect of random fluctuations is also to substitute $N_{\text {bins }}-1$ for $N_{\text {bins }}$ in Eq. (A.3). The proof is completely analogous to the case we just studied. Moreover, it is straightforward to combine the two results and consider minimization with respect to both $b$ and $s$, in which case one should replace $N_{\text {bins }}$ in Eq. (A.3) by $N_{\text {bins }}-2$.

In general, one should use the number of degrees of freedom $N_{\text {d.o.f. when }}$ computing $\left\langle\chi^{2}\right\rangle$ :

$$
\left\langle\chi^{2}\right\rangle=N_{\text {d.o.f. }}+\sum_{i}^{N_{\text {bins }}} \frac{\left(d_{i}-f_{i}\right)^{2}}{d_{i}}
$$

\section{B Analytic Estimate of the Sensitivity Cutoff}

In Sec. 3 we showed that the sensitivity region for anomalous seasonal variations is limited by the finite linewidth of the ${ }^{7} \mathrm{Be}$ line. In this appendix we show how one can analytically estimate the location and the shape of the sensitivity cutoff.

As was mentioned in Sec. 3, the true shape of the ${ }^{7} \mathrm{Be}$ line is rather complicated, with a Gaussian profile on the low end and an exponential tail 
on the high end. For the purpose of this estimate we choose to approximate the Gaussian part by a sharp cutoff:

$$
f(E)=\left\{\begin{array}{ll}
0 & \text { if } E<E_{1} \\
e^{-a E+b} & \text { if } E>E_{1}
\end{array} .\right.
$$

To determine the fraction of neutrinos reaching the Earth we integrate the oscillation probability $P(E, L)$ given by Eq. (3.1) over the line profile Eq. (B.1) and divide by the normalization constant $N$.

$$
\begin{aligned}
\widetilde{P}(L)= & \frac{1}{N} \int d E P(E, L) f(E) \\
\simeq & \frac{1}{N}\left[\left(1-\frac{\sin ^{2} 2 \theta}{2}\right) \int_{E_{1}}^{\infty} d E e^{-a E+b}\right. \\
& \left.+\frac{\sin ^{2} 2 \theta}{2} \int_{E_{1}}^{\infty} d E \cos \left(2 \frac{1.27 \Delta m^{2} L}{E_{0}^{2}}\left(E_{0}-E\right)\right) e^{-a E+b}\right] \\
= & \frac{e^{-a E_{1}+b}}{N}\left[\left(1-\frac{\sin ^{2} 2 \theta}{2}\right) \frac{1}{a}+\frac{\sin ^{2} 2 \theta}{2} \frac{1}{\sqrt{a^{2}+\left(1.27 \Delta m^{2} L / E_{0}^{2}\right)^{2}}}\right. \\
& \left.\times \cos \left(2 \frac{1.27 \Delta m^{2} L}{E_{0}^{2}}\left(E_{0}-E_{1}\right)-\arctan \left(2 \frac{1.27 \Delta m^{2} L}{a E_{0}^{2}}\right)\right)\right] .
\end{aligned}
$$

Since the width of the line is only several $\mathrm{keV}$ while $E_{0}=0.862 \mathrm{MeV}$, we can set $E_{0}-E_{1} \simeq E_{0}$ in the argument of the cosine. Substituting the value of the normalization constant $N=\int_{E_{1}}^{\infty} d E e^{-a E+b}=(1 / a) e^{-a E_{1}+b}$ and introducing $\phi \equiv \arctan \left(2 \times 1.27 \Delta m^{2} L /\left(a E_{0}^{2}\right)\right)$, we obtain

$$
\widetilde{P}(L) \simeq 1-\frac{\sin ^{2} 2 \theta}{2}\left(1-\frac{\cos \left(2 \frac{1.27 \Delta m^{2} L}{E_{0}}-\phi\right)}{\sqrt{1+\left(1.27 \Delta m^{2} L /\left(E_{0}^{2} a\right)\right)^{2}}}\right) .
$$

From this equation we can read off the shape of the cutoff. Viewed as a function of $\Delta m^{2}$, for small values of the mixing angle the cutoff profile is approximately given by

$$
\sin ^{2} 2 \theta_{\text {cutoff }} \propto \sqrt{1+\left(1.27 \Delta m^{2} L /\left(E_{0}^{2} a\right)\right)^{2}} .
$$

Using the numerical value of $a=0.75 \mathrm{keV}^{-1}$, obtained by fitting the line profile in [27, we find that $\sin ^{2} 2 \theta_{\text {cutoff }}\left(\Delta m^{2}\right)$ should increase by $\sqrt{2}$ with respect 
to the smallest value of $\sin ^{2} 2 \theta_{\text {cutoff }}$ when $\Delta m^{2} \simeq 2.9 \times 10^{-9} \mathrm{eV}^{2}$. The actual number from curve 4 in Fig. 7 is $\Delta m^{2} \simeq 1.5 \times 10^{-9} \mathrm{eV}^{2}$. The actual value is smaller, which is expected, because, for the purpose of this estimate, we neglected the contribution of the Gaussian part of the line profile, effectively making the line narrower.

One can also estimate the location of the cutoff if the line profile were purely Gaussian (curve 3 in Fig. 7). The steps are completely analogous: the new normalization constant is $N^{\prime}=\int_{-\infty}^{\infty} d E e^{-\left(E-E_{0}\right)^{2} / \sigma^{2}}=\sqrt{\pi} \sigma$, and $\widetilde{P}(L)$ is given by

$$
\begin{aligned}
\widetilde{P}(L) & =\frac{1}{N^{\prime}} \int_{-\infty}^{\infty} d E P(E, L) e^{-E^{2} / \sigma^{2}} \\
\simeq & \frac{1}{N^{\prime}}\left[\left(1-\frac{\sin ^{2} 2 \theta}{2}\right) \int_{-\infty}^{\infty} d E e^{-E^{2} / \sigma^{2}}\right. \\
& \left.+\frac{\sin ^{2} 2 \theta}{2} \int_{-\infty}^{\infty} d E \cos \left(2 \frac{1.27 \Delta m^{2} L}{E_{0}^{2}}\left(E_{0}-E\right)\right) e^{-E^{2} / \sigma^{2}}\right] \\
= & 1-\frac{\sin ^{2} 2 \theta}{2}\left(1-e^{-\left(1.27 \Delta m^{2} L \sigma / E_{0}^{2}\right)^{2}} \cos \left(2 \frac{1.27 \Delta m^{2} L}{E_{0}}\right)\right) .
\end{aligned}
$$

Thus, the cutoff for this model sets in faster and the profile for small values

of $\sin ^{2} 2 \theta$ is Gaussian. Numerically, $\sin ^{2} 2 \theta_{\text {cutoff }}\left(\Delta m^{2}\right)$ is expected to increase by $\sqrt{2}$ with respect to the smallest value of $\sin ^{2} 2 \theta_{\text {cutoff }}$ when $\Delta m^{2} \simeq 4.2 \times$ $10^{-9} \mathrm{eV}^{2}$, which agrees with curve 3 in Fig. 7 .

\section{References}

[1] Super-Kamiokande Collaboration (Y. Fukuda et al.), Phys. Rev. Lett. 81, 1562 (1998), hep-ex/9807003.

[2] B.T. Cleveland et al., Astrophys. J. 496, 505 (1998), and references therein.

[3] KAMIOKANDE-II Collaboration (K.S. Hirata et al.), Phys. Rev. Lett. 63, 16 (1989).

[4] GALleX Collaboration (W. Hampel et al.), Phys. Lett. B388, 384 (1996); T. Kirsten, talk presented at NEUTRINO '98 conference, June 4-9, 1998, Takayama, Japan. 
[5] SAGE Collaboration (Dzh.N. Abdurashitov et al.), Phys. Lett. B328, 234 (1994); V.N. Gavrin, talk presented at NEUTRINO '98 conference, June 4-9, 1998, Takayama, Japan.

[6] Super-Kamiokande Collaboration (Y. Fukuda et al.), Phys. Rev. Lett. 81, 1158 (1998), hep-ex/9805021.

[7] J.N. Bahcall, P.I. Krastev, and A.Yu. Smirnov, Phys. Rev. D 58, 096016 (1998), hep-ph/9807216.

[8] H. Minakata and H. Nunokawa, Phys. Rev. D 59, 073004 (1999), hep$\mathrm{ph} / 9810387$.

[9] L. Wolfenstein, Phys. Rev. D 17, 2369 (1978).

[10] S.P. Mikheyev and A.Yu. Smirnov, Yad. Fiz. (Sov. J. of Nucl. Phys.) 42, 1441 (1985).

[11] S.L. Glashow and L.M. Krauss Phys. Lett. 190B, 199 (1987).

[12] J.N. Bahcall, Astrophys. J. 467, 475 (1996), hep-ph/9512285.

[13] J.N. Bahcall, Nucl. Phys. A631, 29 (1998), nucl-th/9802050.

[14] V.N. Gribov and B. Pontecorvo, Phys. Lett. 28B, 493 (1969); J.N. Bahcall and S.C. Frautschi, Phys. Lett. 29B, 623 (1969); S.M. Bilenky and B. Pontecorvo, Phys. Rep. 41C, 225 (1978); R. Ehrlich, Phys. Rev. D 18, 2323 (1978); R. Barbieri, J. Ellis, and M.K. Gaillard, Phys. Lett. 90B, 249 (1980); K. Whisnant, R. Phillips, and V. Barger, Phys. Rev. D 24, 538 (1981).

[15] S. Pakvasa and J. Pantaleone, Phys. Rev. Lett. 65, 2479 (1990).

[16] V. Barger, R.J.N. Phillips, and K. Whisnant, Phys. Rev. Lett. 65, 3084 (1990); Z.G. Berezhiani, A. Rossi, Phys. Rev D 51, 5229 (1995), hep-ph/9409464; P.I. Krastev and S.T. Petcov, Nucl. Phys. B449, 605 (1996), hep-ph/9408234; B. Faïd, G.L. Fogli, E. Lisi, D. Montanino, Phys. Rev. D 55, 1353 (1997), hep-ph/9608311; Astropart. Phys. 10, 93 (1999), hep-ph/9805293; J.M. Gelb and S.P. Rosen, hep-ph/9809508;

V. Berezinsky, G. Fiorentini, and M. Lissia, INFN-CA-TH-98-15, hep$\mathrm{ph} / 9811352$. 
[17] S.L. Glashow, P.J. Kernan, and L.M. Krauss, Phys. Lett. B445, 412 (1999), hep-ph/9808470; V. Barger and K. Whisnant, MADPH-991107, hep-ph/9903262; M. Maris and S.T. Petcov, SISSA-13-99-EP, hepph/9903303.

[18] L. Oberauer, talk presented at NEUTRINO '98 conference, June 4-9, 1998, Takayama, Japan; J.B. Benziger et al., "A Proposal for Participation in the Borexino Solar Neutrino Experiment", http://pupgg.princeton.edu/ Borexino/ppp.htm.

[19] A. Suzuki, talk presented at NEUTRINO '98 conference, June 4-9, 1998, Takayama, Japan.

[20] J. Busenitz et al., "Proposal for US Participation in KamLAND," March 1999 (unpublished).

[21] A possible way of experimentally obtaining this information was proposed recently by two of the authors in A. de Gouvêa and H. Murayama, Phys. Rev. Lett. 82, 3392 (1999), hep-ph/9812307.

[22] J.N. Bahcall and M.H. Pinsonneault, Rev. Mod. Phys. 67, 781 (1995), hep-ph/9505425.

[23] J.N. Bahcall, S. Basu, M.H. Pinsonneault, Phys. Lett. B433, 1 (1998), astro-ph/9805135.

[24] J. Pantaleone, Phys. Lett. B251, 618 (1990).

[25] S.T. Petcov, Phys. Lett. B200, 373 (1988); P.I. Krastev and S.T. Petcov, Phys. Lett. B207, 64 (1988); Erratum, ibid., B214, 661 (1988); S.T. Petcov, Phys. Lett. B214, 139 (1988);

[26] J.N. Bahcall, "Neutrino Astrophysics," Cambridge University Press, 1989.

[27] J.N. Bahcall, Phys. Rev. D 49, 3923 (1994).

[28] A. Loeb, Phys. Rev. D 39, 1009 (1989).

[29] G.L. Fogli, E. Lisi, D. Montanino, Phys. Rev. D 56, 4374 (1997), hepph/9706228. 
[30] M. Smy, talk at the American Physical Society (APS) Meeting of the Division of Particles and Fields (DPF 99), Los Angeles, CA, Jan 5-9, 1999, hep-ex/9903006.

[31] A. McDonald, talk presented at NEUTRINO '98 conference, June 4-9, 1998, Takayama, Japan. 